\title{
Mass transport in water waves over a thin layer of soft viscoelastic mud
}

\author{
CHIU-ON NG AND XUEYAN ZHANG \\ Department of Mechanical Engineering, The University of Hong Kong, \\ Pokfulam Road, Hong Kong
}

(Received 30 December 2005 and in revised form 2 August 2006)

A theory is presented for the mass transport induced by a small-amplitude progressive wave propagating in water over a thin layer of viscoelastic mud modelled as a Voigt medium. Based on a sharp contrast in length scales near the bed, the boundary-layer approximation is applied to the Navier-Stokes equations in Lagrangian form, which are then solved for the first-order oscillatory motions in the mud and the near-bed water layers. On extending the analysis to second order for the mass transport, it is pointed out that it is inappropriate, as was done in previous studies, to apply the complex viscoelastic parameter to a higher-order analysis, and also to suppose that a Voigt body can undergo continuous steady motion. In fact, the time-mean motion of a Voigt body is only transient, and will stop after a time scale given by the ratio of the viscosity to the shear modulus. Once the mud has attained its steady deformation, the mass transport in the overlying water column can be found as if it were a single-layer system. It is found that the near-bed mass transport has non-trivial dependence on the mud depth and elasticity, which control the occurrence of resonance. Even when the resonance is considerably damped by viscosity, the mass transport in water over a viscoelastic layer can be dramatically different, in terms of magnitude and direction, from that over a rigid bed.

\section{Introduction}

Wave-mud interaction is one of the key mechanisms controlling the transport of sediments in coastal and estuarine waters. In the presence of cohesive sediments or marine muds, which are composed primarily of very fine particles and act as an effective energy dissipator, wave damping is enhanced; surface waves can be attenuated appreciably in a finite number of wave periods or wavelengths. Frequency modulation may also occur to waves propagating over a muddy bottom. Meanwhile, the sediments will undergo various processes such as resuspension, fluidization and mass transport under the forcing of surface waves. The erosion, transport and deposition of cohesive sediments are known to pose various problems that are of concern to the environmental as well as coastal engineering. These problems have motivated extensive studies into the mechanics of mud in a wave-dominated marine environment.

Cohesive sediments are made up of very tiny particles, typically less than $O(10) \mu \mathrm{m}$ in size, and their properties are much determined by the micro-structure which is dominated by many physico-chemical effects. Since the mineralogical make-up is so complex and susceptible to change in response to the local geo-environment, a mud tends to exhibit vastly different rheological behaviours depending on the site conditions. For the sake of analysis, it is common in the modelling of wave-seabed 
interaction that the bottom material is treated simply as a linear medium: either a Newtonian viscous fluid (e.g. Dalrymple \& Liu 1978; Ng 2000), or a linear elastic soil (e.g. Mallard \& Dalrymple 1977; Wen \& Liu 1995a). Experimental observations, however, reveal that a wave-fluidized mud can be highly non-Newtonian (e.g. Maa \& Mehta 1988; Verreet \& Berlamont 1988; Chou, Foda \& Hunt 1993). Using a nonlinear rheological model based on their laboratory measurements, Foda, Hunt \& Chou (1993) performed a theoretical study on the fluidization of marine mud under waves. Their experiments demonstrate that a cohesive mud will respond to oscillatory shear according to a nonlinear function of the strain. There is a lower limit of elastic yield strain, below which the mud behaves as an elastic solid, and an upper limit of viscous critical strain, above which the mud behaves as a pure viscous fluid. For an intermediate strain amplitude between these two limits, the mud exhibits both viscous and elastic behaviours and can be described as a viscoelastic material.

These three response regimes correspond to the three states in which a cohesive sediment may exist in nature (Whitehouse et al. 2000): it is viscous when completely fluidized to form the so-called fluid mud; it is viscoelastic when freshly deposited to form a partially consolidated bed; and it is elastic when completely settled to form a consolidated bed. In other words, the superficial sediment in which particles are fluid-supported tends to behave more like a viscous fluid, while the deeper sediment that is consolidated behaves more like an elastic solid. To describe this stratification structure, it is desirable, as far as modelling is concerned, that the cohesive mud be generalized into a material possessing both viscous and elastic characteristics. In this connection, the viscoelastic Voigt model has been increasingly adopted in studies on the wave-mud interaction, as in Hsiao \& Shemdin (1980), MacPherson (1980), Maa \& Mehta (1990), Piedra-Cueva (1993), and Hill \& Foda (1999). In particular, Maa \& Mehta (1990) pointed out the possibility of resonance of the system arising from the viscoelasticity, and Piedra-Cueva (1993) found that the wave damping may be enhanced by some orders of magnitude when resonance occurs. In these previous studies, the focus is on the effects of viscoelasticity on the wave transformation, and therefore it suffices to consider only the first-order oscillatory motion.

The Voigt model, which has been considered to describe viscoelastic muds better than the Maxwell model (Maa \& Mehta 1988), has been commonly adopted in the literature. The constitutive equation for a Voigt body under simple shear can be written as

$$
\tau=\rho \nu \dot{\gamma}+G \gamma,
$$

where $\tau$ is shear stress, $\gamma$ and $\dot{\gamma}$ are, respectively, the strain and strain rate, $G$ is the elastic modulus, $\rho$ is the density, and $v$ is the kinematic viscosity. If the disturbance is further assumed to vary sinusoidally in time, such as $\mathrm{e}^{-\mathrm{i} \sigma t}$, where $\mathrm{i}$ is the complex unit, $\sigma$ is the oscillation frequency, and $t$ is the time, then the strain and strain rate can be related to each other by

$$
\dot{\gamma}=-\mathrm{i} \sigma \gamma,
$$

and the constitutive equation can be simplified to

$$
\tau=\rho v^{\dagger} \dot{\gamma},
$$

where $v^{\dagger}$ is a complex parameter which conveniently combines the viscosity and elasticity and can be expressed as

$$
v^{\dagger}=v+\mathrm{i} G / \rho \sigma .
$$


The introduction of the complex viscoelastic parameter allows an analysis for a viscoelastic medium to be performed in exactly the same manner as that for a pure viscous fluid. This approach has been extensively followed, as in Tchen (1956), MacPherson (1980), Hsiao \& Shemdin (1980), and Piedra-Cueva (1993).

We emphasize that the complex viscoelastic parameter, as introduced in (1.4), is applicable only to a motion that is simple harmonic. Such a limitation seems not to have been appreciated by some workers. For example, Shibayama, Okuno \& Sato (1990), Lian, Zhao \& Zhang (1999) and Soltanpour, Shibayama \& Noma (2003) have used the viscoelastic parameter to determine the second-order mean drift (or mass transport) induced by waves in a layer of viscoelastic mud. Such an application of the viscoelastic parameter is obviously questionable, since the second-order motion comprises higher harmonic and steady components, for which the strain and strain rate are no longer related to each other by (1.2). Moreover, a viscoelastic material will behave differently from a viscous fluid when subjected to a steady load. For a material possessing elasticity and viscosity in parallel, the shear deformation in response to a constant shear stress cannot go on continuously, but will stop once a finite limit is attained. Therefore, a steady particle drift cannot be sustained in a Voigt material. This phenomenon has been examined and confirmed theoretically by Zhang \& $\mathrm{Ng}(2006 b)$.

Mass transport, also known as Lagrangian or particle drift, is a time-mean current induced in a wave boundary layer owing to the convective inertia of the waves. It is therefore a quantity of second order in wave steepness. Longuet-Higgins (1953) presented a detailed exposition of the basic theory of mass transport in water waves over a rigid impermeable bed. Since then, many works have studied the effects of a non-rigid or deformable bed on the mass transport in the water column. Examples are: mass transport in a two-layer viscous system (Dore 1970; Piedra-Cueva 1995; Wen \& Liu 1995b; Ng 2004b), in water over a finite layer of non-Newtonian mud (Sakakiyama \& Bijker 1989; Isobe, Huynh \& Watanabe 1992; Ng, Fu \& Bai 2002), in water over a porous bed (Liu 1977), and in water over an infinitely deep elastic medium (Wen \& Liu 1995a).

Although viscoelasticity is increasingly recognized as an important property of a muddy bed, and has been extensively studied for its first-order effects on the waves, its effects on the second-order mass transport in waves are by contrast much less studied. Some workers, such as Shibayama et al. (1990), Lian et al. (1999) and Soltanpour et al. (2003), have attempted to work out the transport rate of viscoelastic mud under wave action, but, as pointed out above, they have inappropriately applied the complex viscoelastic parameter to the second-order problem. Despite these studies, no work in the literature has specifically examined mass transport in water waves over a viscoelastic bed. This forms the motivation and objective of the present study.

This paper aims to present a theory of mass transport in water waves in a two-layer system, in which the upper layer is clear water and the lower layer is a thin layer of soft viscoelastic mud modelled as a Voigt medium. The key assumptions are as follows. (i) Conditions corresponding to a mild wave environment or slack water are supposed to prevail so that the bed materials will only be subjected to small-amplitude stresses, well below that for plastic yield. As a result, a linear viscoelastic model with constant coefficients can be applied here. (ii) The environment is also conducive to the formation of a lutocline (i.e. strong gradient in concentration), or a persistent sharp interface between the water column and the muddy bed. Across the lutocline, the dry mass concentration may change abruptly from $O(1) \mathrm{kg} \mathrm{m}^{-3}$ in the water column to $O(100) \mathrm{kg} \mathrm{m}^{-3}$ in the near-bed fluid mud (Mehta 1996). As remarked by Winterwerp (1999), any suspension of intermediate concentrations can only be transient and will 
not be stable, and a jump may persist in the density profile (see also Whitehouse et al. 2000). The sharp density gradient causes the Richardson number to exceed amply the critical value required for stable stratification, even under some wave agitation. Consequently, turbulence is largely damped out on crossing the interface, and therefore the entrainment of fluid mud into the overlying water is suppressed. The mud flow, if any, is dominated by molecular viscous force, while water can be turbulent in the boundary layer above the interface. The condition under which interfacial mixing is insignificant for waves over a mud with yield stress has been examined by Mei \& Liu (1987). (iii) The viscoelastic layer in the present problem is to represent a layer of partially fluidized mud or under-consolidated soil with weak elasticity resulting from dewatering of the fluid mud. A more realistic model, such as Chou et al. (1993), should of course have the system divided into several discrete layers: the water column, viscous fluid-like mud, viscoelastic soft mud, and elastic stiff mud, where the depths of the fluid and soft mud layers are not known a priori and are found as part of the solution depending on the hydrodynamic forcing. Such a nonlinear approach is, however, algebraically and computationally intensive and is not followed here. Instead, we consider a simplified stratification structure, in which a clear water column is underlain by a single viscoelastic mud layer resting on a rigid bottom, where the mud depth and properties are prescribed constants.

At slack water, there is a strong tendency for the fluid mud to dewater and consolidate to form a weak soil when the stress falls below $0.1 \mathrm{~N} \mathrm{~m}^{-2}$ (Whitehouse et al. 2000). Li \& Mehta (2001) remarked that in the density range of $1200-1300 \mathrm{~kg} \mathrm{~m}^{-3}$, a fluid mud that has virtually no elasticity or shear strength, in the absence of drastic agitation, will develop into a weakly elastic particulate matrix with a measurable shear strength. In the present problem, the fluidized mud is taken to be a weak soil at most, possessing only the lowest measurable viscoelastic strength, namely, the viscosity in Pa s, and the elastic modulus in Pa, are both of $O(10)$ (Mehta 1996).

The fluidized mud is typically $O(10) \mathrm{cm}$ deep, as has been reported in many field and laboratory studies (e.g. Mehta 1996; Rodriguez \& Mehta 1998). This mud thickness is comparable with the surface-wave amplitude under fair weather conditions. Also, for a gravity wave of frequency $\sigma=O(1) \mathrm{s}^{-1}$ propagating in a medium of density $\rho_{m}=$ $O\left(10^{3}\right) \mathrm{kg} \mathrm{m}^{-3}$, viscosity $\mu_{m}=O(10) \mathrm{Pas}$ and elastic modulus $G_{m}=O(10) \mathrm{Pa}$, the thickness of the oscillatory (or Stokes) boundary layer, $\left(2 \mu_{m} / \rho_{m} \sigma\right)^{1 / 2}$, and the wavelength of induced elastic waves, $\left(G_{m} / \rho_{m} \sigma^{2}\right)^{1 / 2}$, are both of $O(10) \mathrm{cm}$. It is therefore assumed in the present model that the mud depth, the mud Stokes boundary-layer thickness, and the wavelength of elastic waves are all comparable with the surface-wave amplitude, which in turn is much smaller than the surface wavelength and the water depth. Based on this assumption of sharp contrast in length scales, the two-layer Stokes boundary-layer model of $\mathrm{Ng}$ (2000) can be invoked. The first-order oscillatory solutions valid within the entire viscoelastic layer and in the interfacial boundary layer of water are first worked out. Second-order solutions are then developed to determine the mass transport velocity field across the entire (i.e. the surface and bottom boundary layers and the core region) water layer. To simplify the analysis, we may consider an instant of time at which the viscoelastic mud has already stopped undergoing mean motion, or the mean deformation of the mud has already attained its equilibrium state. In other words, the focus here is primarily on the mass transport in the water layer, and the objective is to find out how it is affected by the viscoelastic oscillatory motion of a compliant mud on the bed.

Following Ünlüata \& Mei (1970), Piedra-Cueva (1995), and Ng (2004a), we base our analysis on the Lagrangian description. The use of Lagrangian coordinates will 


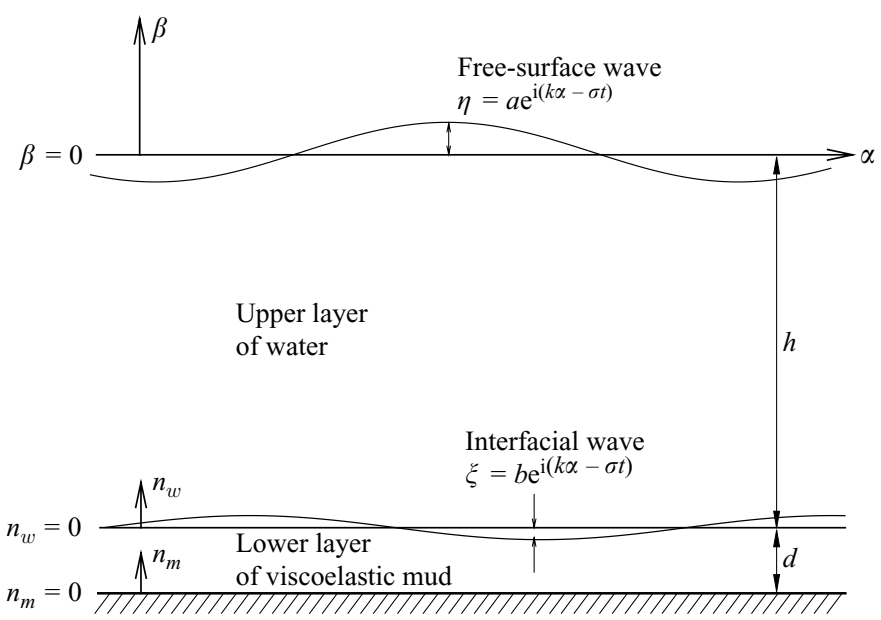

Figure 1. Definition sketch of the problem.

enable boundary conditions to be specified exactly on the moving boundaries (i.e. the free-surface and the water-mud interface) rather than their mean levels as in an Eulerian analysis. This requirement must be fulfilled in order to cater for the condition that the displacement amplitude of these moving boundaries is usually much larger than the boundary-layer thickness. If Eulerian coordinates are used instead, and the boundary conditions are to be specified upon a Taylor expansion about the mean level of the moving boundaries, the solutions so obtained will be limited to waves of a very small amplitude (Longuet-Higgins 1953). The Lagrangian approach is alternative to the curvilinear coordinate system introduced by Longuet-Higgins (1953). Also, mass transport and material deformations are by definition Lagrangian quantities, and it will be more natural and convenient if a Lagrangian formulation is used directly. Monin \& Yaglom (1971) can be consulted for a derivation of the Navier-Stokes equations in Lagrangian form.

\section{Basic formulation}

As in figure 1, we consider a two-layer system in which a layer of clear water overlies a thin layer of viscoelastic mud; the depths of the two layers are $h$ and $d$, respectively, where $h \gg d$. As discussed above, it is assumed that stable stratification prevails, and the two layers are separated by a sharp interface. We refer the equations of motion to Lagrangian coordinates $(\alpha, \beta)$, which are the undisturbed horizontal/vertical positions of a material particle. The $\alpha$ - and $\beta$-axes are, respectively, directed along the surfacewave propagation, and vertically upward from the equilibrium level of the free water surface. The instantaneous position of a particle, denoted by $(x, z)$, is a function of $\alpha$, $\beta$ and $t \geqslant 0$. We also introduce, for the convenience of expressing the boundary-layer solutions to be developed below, two local boundary-layer coordinates $n_{m} \equiv \beta+h+d$ and $n_{w} \equiv \beta+h=n_{m}-d$, which point upward from the bottom of the mud layer, and from the water-mud interface, respectively (figure 1). Two distinct local coordinates are required, since the Stokes boundary-layer thicknesses in the two layers are in general of disparate orders of magnitude (see below). 
A train of small-amplitude progressive waves propagates on the free surface $\beta=0$, whose displacement is given by

$$
\eta(\alpha, t)=a \mathrm{e}^{\mathrm{i}(k \alpha-\sigma t)},
$$

where $a$ is the wave amplitude, $k$ is the wavenumber, and $\sigma$ is the angular frequency. Moderate wavelengths are considered such that $k h=O(1)$. The mud depth $d$, and the Stokes boundary-layer thickness in the mud $\delta_{m} \equiv\left(2 v_{m} / \sigma\right)^{1 / 2}$, where $v_{m}=\mu_{m} / \rho_{m}$ is the kinematic viscosity of the mud, are both of the same order of magnitude as the surface-wave amplitude $a$, which is much smaller than the wavelength. Two comparable small parameters can be defined as follows:

$$
\varepsilon \equiv k a \sim \varepsilon_{m} \equiv k \delta_{m} \ll 1,
$$

where $\varepsilon$ is the wave steepness. The mud layer is so shallow that the entire layer is driven by viscous forcing.

Immediately above the water-mud line, the water is also subject to boundarylayer viscous flow, which can be laminar or turbulent depending on the near-bottom Reynolds number. If turbulent, the mixing coefficient, or eddy viscosity, is known to vary with depth and time in a wave boundary layer. We, however, follow Mei (1989, p. 420) and adopt the assumption of constant eddy viscosity, based on the argument that the varying component of the eddy viscosity should not have significant effects on the flow under consideration. It has been reported that under long waves, the temporal variation of the eddy viscosity may lead to dramatic change to the second-order flow field (Trowbridge \& Madsen 1984); this aspect is ignored in the present study.

The value of eddy viscosity is scale dependent, and for a typical seabed in a mild wave environment, the eddy viscosity can be as much as $v_{w}=O(1) \mathrm{cm}^{2} \mathrm{~s}^{-1}$, i.e. 100 times the molecular viscosity. Accordingly, the Stokes boundary-layer thickness in water is given by $\delta_{w}=\left(2 v_{w} / \sigma\right)^{1 / 2}=O(1) \mathrm{cm}$, which is smaller than that in the mud, $\delta_{m}=O(10) \mathrm{cm}$, as has been estimated above. A third small parameter can be defined in terms of $\delta_{w}$ :

$$
\varepsilon_{w} \equiv k \delta_{w} \ll 1 \text {. }
$$

It follows that, for both the mud and the near-bottom water, we may apply the boundary-layer approximation to the equations of motion based the following scalings of the variables:

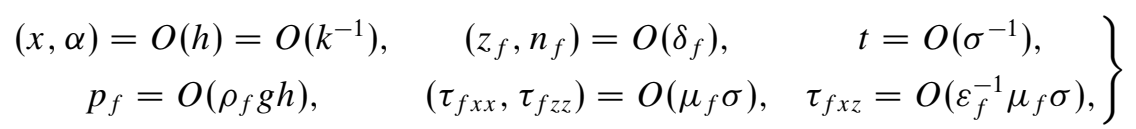

where $\rho$ is the density, $\mu=\rho v$ is the dynamic viscosity, $p$ is the pressure, and $\tau_{i j}$ are the stress components. The subscript $f=(w, m)$ is used to denote the association of a quantity with the water or mud, or to distinguish between the two material domains

$$
f=\left\{\begin{array}{ll}
w & \text { for water in } n_{w}>0 \\
m & \text { for mud in } d>n_{m}>0 .
\end{array} .\right.
$$

The two local coordinates are scaled by the corresponding Stokes boundary-layer thicknesses.

The Navier-Stokes equations in Lagrangian form, which are obtainable from Pierson (1962), Monin \& Yaglom (1971) and Piedra-Cueva (1995), are expressible as follows, in which the small parameter $\varepsilon_{f}=k \delta_{f}$ is inserted for identification of the order of the subdominant terms. Using the standard notation for a Jacobian, the 
conservation of mass is governed by

$$
\frac{\partial\left(x_{f}, z_{f}\right)}{\partial\left(\alpha, n_{f}\right)}=1,
$$

and the horizontal and vertical momentum equations are

$$
\begin{aligned}
\frac{\partial^{2} x_{f}}{\partial t^{2}} & =-\frac{1}{\rho_{f}} \frac{\partial\left(p_{f}, z_{f}\right)}{\partial\left(\alpha, n_{f}\right)}+\frac{1}{\rho_{f}}\left[\varepsilon_{f}^{2} \frac{\partial\left(\tau_{f x x}, z_{f}\right)}{\partial\left(\alpha, n_{f}\right)}+\frac{\partial\left(x_{f}, \tau_{f x z}\right)}{\partial\left(\alpha, n_{f}\right)}\right], \\
\varepsilon_{f}^{2} \frac{\partial^{2} z_{f}}{\partial t^{2}} & =-\frac{1}{\rho_{f}} \frac{\partial\left(x_{f}, p_{f}\right)}{\partial\left(\alpha, n_{f}\right)}+\frac{\varepsilon_{f}^{2}}{\rho_{f}}\left[\frac{\partial\left(\tau_{f z x}, z_{f}\right)}{\partial\left(\alpha, n_{f}\right)}+\frac{\partial\left(x_{f}, \tau_{f z z}\right)}{\partial\left(\alpha, n_{f}\right)}\right]-\varepsilon_{f} g .
\end{aligned}
$$

Here we assume that the viscoelastic mud is an isotropic Voigt body, for which the stress is a linear combination of viscous and elastic parts, where the elastic part of the stress is linearly proportional to the Lagrangian finite-strain tensor (e.g. Lai, Rubin \& Krempl 1993; Hutter \& Jöhnk 2004):

$$
E_{i j}=\frac{\partial u_{i}}{\partial X_{j}}+\frac{\partial u_{j}}{\partial X_{i}}+\frac{\partial u_{m}}{\partial X_{i}} \frac{\partial u_{m}}{\partial X_{j}},
$$

where $u_{i}$ is the displacement vector of a particle that is originally positioned at $X_{i}$. It follows that we may express the Lagrangian stress components as follows:

$$
\begin{aligned}
\tau_{f x x}= & 2 \mu_{f} \frac{\partial\left(\dot{x}_{f}, z_{f}\right)}{\partial\left(\alpha, n_{f}\right)}+G_{f}\left[2 \frac{\partial\left(x_{f}-\alpha\right)}{\partial \alpha}+\frac{\partial\left(x_{f}-\alpha\right)}{\partial \alpha} \frac{\partial\left(x_{f}-\alpha\right)}{\partial \alpha}\right. \\
& \left.+\varepsilon_{f}^{2} \frac{\partial\left(z_{f}-n_{f}\right)}{\partial \alpha} \frac{\partial\left(z_{f}-n_{f}\right)}{\partial \alpha}\right], \\
\tau_{f z z}= & 2 \mu_{f} \frac{\partial\left(x_{f}, \dot{z}_{f}\right)}{\partial\left(\alpha, n_{f}\right)}+G_{f}\left[2 \frac{\partial\left(z_{f}-n_{f}\right)}{\partial n_{f}}+\frac{1}{\varepsilon_{f}^{2}} \frac{\partial\left(x_{f}-\alpha\right)}{\partial n_{f}} \frac{\partial\left(x_{f}-\alpha\right)}{\partial n_{f}}\right. \\
& \left.+\frac{\partial\left(z_{f}-n_{f}\right)}{\partial n_{f}} \frac{\partial\left(z_{f}-n_{f}\right)}{\partial n_{f}}\right],
\end{aligned}
$$

and

$$
\begin{aligned}
\tau_{f x z}=\tau_{f z x}= & \mu_{f}\left[\frac{\partial\left(x_{f}, \dot{x}_{f}\right)}{\partial\left(\alpha, n_{f}\right)}+\varepsilon_{f}^{2} \frac{\partial\left(\dot{z}_{f}, z_{f}\right)}{\partial\left(\alpha, n_{f}\right)}\right]+G_{f}\left[\frac{\partial\left(x_{f}-\alpha\right)}{\partial n_{f}}+\varepsilon_{f}^{2} \frac{\partial\left(z_{f}-n_{f}\right)}{\partial \alpha}\right. \\
& \left.+\frac{\partial\left(x_{f}-\alpha\right)}{\partial \alpha} \frac{\partial\left(x_{f}-\alpha\right)}{\partial n_{f}}+\varepsilon_{f}^{2} \frac{\partial\left(z_{f}-n_{f}\right)}{\partial \alpha} \frac{\partial\left(z_{f}-n_{f}\right)}{\partial n_{f}}\right]
\end{aligned}
$$

where the overdot denotes time derivative, $G_{w}=0$ for water is a pure Newtonian fluid, and $G_{m}$ is the modulus of rigidity of mud.

The boundary and matching conditions are as follows. The no-slip at the solid bottom requires that

$$
x_{m}=z_{m}=\dot{x}_{m}=\dot{z}_{m}=0 \quad \text { on } n_{m}=0 .
$$

The water-mud interface, $n_{m}=d$, will have a displacement $\xi(\alpha, t)$ that is expected to be an order of magnitude smaller in amplitude than the free-surface wave. The continuity of particle displacements and stress components along this interface gives

$$
\begin{gathered}
x_{m}=x_{w}, \quad z_{m}-d=z_{w}=\xi \quad \text { on } n_{m}=d \text { or } n_{w}=0, \\
T_{m}=T_{w}, \quad N_{m}=N_{w} \quad \text { on } n_{m}=d \text { or } n_{w}=0,
\end{gathered}
$$


where $T$ and $N$ are, respectively, the tangential and normal stress components on a material surface (Piedra-Cueva 1995):

$$
\begin{aligned}
T_{f}= & \varepsilon_{f}^{2}\left(\tau_{f z z}-\tau_{f x x}\right) \frac{\partial x_{f}}{\partial \alpha} \frac{\partial z_{f}}{\partial \alpha}+\tau_{f z x}\left[\left(\frac{\partial x_{f}}{\partial \alpha}\right)^{2}-\varepsilon_{f}^{2}\left(\frac{\partial z_{f}}{\partial \alpha}\right)^{2}\right], \\
N_{f}= & -p_{f}\left[\left(\frac{\partial x_{f}}{\partial \alpha}\right)^{2}+\varepsilon_{f}^{2}\left(\frac{\partial z_{f}}{\partial \alpha}\right)^{2}\right] \\
& +\varepsilon_{f}^{2}\left[\varepsilon_{f}^{2} \tau_{f x x}\left(\frac{\partial z_{f}}{\partial \alpha}\right)^{2}+\tau_{f z z}\left(\frac{\partial x_{f}}{\partial \alpha}\right)^{2}-2 \tau_{f x z} \frac{\partial x_{f}}{\partial \alpha} \frac{\partial z_{f}}{\partial \alpha}\right] .
\end{aligned}
$$

The particle displacements are primarily caused by a perturbation of order $\varepsilon=k a$, and therefore the variables can be expanded into powers of $\varepsilon$ :

$$
\begin{gathered}
\left(x_{f}, z_{f}\right)=\left(\alpha, n_{f}\right)+\varepsilon\left(x_{f 1}, z_{f 1}\right)+\varepsilon^{2}\left(x_{f 2}, z_{f 2}\right)+\cdots, \\
p_{f}=\rho_{w} g h-\varepsilon_{f} \rho_{f} g\left(n_{m}-d\right)+\varepsilon p_{f 1}+\varepsilon^{2} p_{f 2}+\cdots, \\
(\eta, \xi)=\varepsilon\left(\eta_{1}, \xi_{1}\right)+\varepsilon^{2}\left(\eta_{2}, \xi_{2}\right)+\cdots, \\
\tau_{f z z}=\tau_{f z z 0}+\varepsilon \tau_{f z z 1}+\cdots, \\
\left(\tau_{f x z}, \tau_{f x x}\right)=\varepsilon\left(\tau_{f x z 1}, \tau_{f x x 1}\right)+\varepsilon^{2}\left(\tau_{f x z 2}+\tau_{f x x 2}\right)+\cdots,
\end{gathered}
$$

where

$$
\begin{gathered}
\tau_{f z z 0}=G_{f}\left(\frac{\varepsilon}{\varepsilon_{f}}\right)^{2}\left(\frac{\partial x_{f 1}}{\partial n_{f}}\right)^{2} \\
\tau_{f z z 1}=2 \mu_{f} \frac{\partial \dot{z}_{f 1}}{\partial n_{f}}+2 G_{f}\left[\frac{\partial z_{f 1}}{\partial n_{f}}+\left(\frac{\varepsilon}{\varepsilon_{f}}\right)^{2} \frac{\partial x_{f 1}}{\partial n_{f}} \frac{\partial x_{f 2}}{\partial n_{f}}\right] \\
\tau_{f x z 1}=\mu_{f} \frac{\partial \dot{x}_{f 1}}{\partial n_{f}}+G_{f} \frac{\partial x_{f 1}}{\partial n_{f}} \\
\tau_{f x z 2}=\mu_{f}\left(\frac{\partial \dot{x}_{f 2}}{\partial n_{f}}+\frac{\partial x_{f 1}}{\partial \alpha} \frac{\partial \dot{x}_{f 1}}{\partial n_{f}}-\frac{\partial x_{f 1}}{\partial n_{f}} \frac{\partial \dot{x}_{f 1}}{\partial \alpha}\right)+G_{f}\left(\frac{\partial x_{f 2}}{\partial n_{f}}+\frac{\partial x_{f 1}}{\partial \alpha} \frac{\partial x_{f 1}}{\partial n_{f}}\right), \\
\tau_{f x x x 1}=2 \mu_{f} \frac{\partial \dot{x}_{f 1}}{\partial \alpha}+2 G_{f} \frac{\partial x_{f 1}}{\partial \alpha}, \\
2 \mu_{f}\left(\frac{\partial \dot{x}_{f 2}}{\partial \alpha}+\frac{\partial \dot{x}_{f 1}}{\partial \alpha} \frac{\partial z_{f 1}}{\partial n_{f}}-\frac{\partial \dot{x}_{f 1}}{\partial n_{f}} \frac{\partial z_{f 1}}{\partial \alpha}\right)+G_{f}\left[2 \frac{\partial x_{f 2}}{\partial \alpha}+\left(\frac{\partial x_{f 1}}{\partial \alpha}\right)^{2}\right] .
\end{gathered}
$$

Let us recall the relationships between the three small parameters, as estimated earlier:

$$
\varepsilon_{w} \ll \varepsilon_{m} \sim \varepsilon \ll 1 .
$$

The existence of two disparate small parameters in the water boundary layer would, in principle, require the water variables to be further expanded in terms of $\varepsilon_{w}$. For 
example,

$$
\left(x_{w 1}, z_{w 1}\right)=\left(x_{w 1,0}, z_{w 1,0}\right)+\varepsilon_{w}\left(x_{w 1,1}, z_{w 1,1}\right)+\cdots,
$$

and so on. However, as pointed out by Wen \& Liu (1995a), the terms of $O\left(\varepsilon \varepsilon_{w}\right)$ can be safely ignored, since neglecting these terms will cause only insignificant errors of $O\left(\varepsilon^{2} \varepsilon_{w}\right)$ in the mass transport problem of $O\left(\varepsilon^{2}\right)$.

Now, upon substitution of the expansions (2.18)-(2.22) into the equations of motion (2.6)-(2.8) and boundary conditions (2.13)-(2.15), and collection of terms of powers of $\varepsilon$ and $\varepsilon^{2}$, we may obtain the first- and second-order problems as detailed below.

\section{First-order solutions}

At $O(\varepsilon)$, the continuity and momentum equations are

$$
\begin{gathered}
\frac{\partial x_{f 1}}{\partial \alpha}+\frac{\partial z_{f 1}}{\partial n_{f}}=0 \\
\frac{\partial^{2} x_{f 1}}{\partial t^{2}}=-\frac{1}{\rho_{f}} \frac{\partial p_{f 1}}{\partial \alpha}+\frac{1}{\rho_{f}} \frac{\partial \tau_{f x z 1}}{\partial n_{f}}, \\
0=-\frac{1}{\rho_{f}} \frac{\partial p_{f 1}}{\partial n_{f}},
\end{gathered}
$$

where $\tau_{f x z 1}$ is given by (2.25).

The first-order boundary and matching conditions are

$$
\begin{gathered}
x_{m 1}=z_{m 1}=0 \quad \text { on } n_{m}=0, \\
x_{m 1}=x_{w 1}, \quad z_{m 1}=z_{w 1}=\xi_{1} \quad \text { on } n_{m}=d \text { or } n_{w}=0, \\
\tau_{m x z 1}=\tau_{w x z 1}, \quad p_{m 1}=p_{w 1} \quad \text { on } n_{m}=d \text { or } n_{w}=0 .
\end{gathered}
$$

At the outer edge of the water boundary layer, the particle displacements and pressure are asymptotically equal to the near-bottom values of the core flow:

$$
\left(x_{w 1}, p_{w 1}\right) \rightarrow\left(X_{I}, P_{I}\right) \quad \text { as } n_{w} \gg \delta_{w}, \beta \rightarrow-h,
$$

where the near-bottom core flow is governed by

$$
\frac{\partial^{2} X_{I}}{\partial t^{2}}=-\frac{1}{\rho_{w}} \frac{\partial P_{I}}{\partial \alpha} \quad \text { as } \beta \rightarrow-h .
$$

It is clear from (3.3) and the matching and boundary conditions that $p_{f 1}$, which is the leading-order dynamic pressure, is constant across the layers and equal to

$$
p_{f 1}=P_{I} \text {. }
$$

Therefore, on using (3.8), (3.2) can be written as

$$
\frac{\partial^{2} x_{f 1}}{\partial t^{2}}=\frac{\rho_{w}}{\rho_{f}} \frac{\partial^{2} X_{I}}{\partial t^{2}}+\frac{1}{\rho_{f}} \frac{\partial \tau_{f x z 1}}{\partial n_{f}} .
$$

Let us now express the first-order quantities in the same harmonic form as the free-surface wave

$$
\left(x_{f 1}, z_{f 1}, p_{f 1}, X_{I}, \xi_{1}\right)=\left(\tilde{x}_{f}, \tilde{z}_{f}, \tilde{p}_{f}, \tilde{X}_{I}, b\right) \mathrm{e}^{\mathrm{i}(k \alpha-\sigma t)},
$$

where $\tilde{x}_{f}=\tilde{x}_{f}\left(n_{f}\right), \tilde{z}_{f}=\tilde{z}_{f}\left(n_{f}\right)$ and $\tilde{p}_{f}=\tilde{p}_{f}\left(n_{f}\right)$ are complex functions of $n_{f}$ only. 
Equation (3.10) then yields, for the two domains,

$$
\begin{gathered}
\frac{\mathrm{d}^{2} \tilde{x}_{w}}{\mathrm{~d} n_{w}^{2}}=-\frac{\mathrm{i} \sigma}{v_{w}}\left(\tilde{x}_{w}-\tilde{X}_{I}\right) \quad \text { in } n_{w}>0, \\
\frac{\mathrm{d}^{2} \tilde{x}_{m}}{\mathrm{~d} n_{m}^{2}}=-\frac{\mathrm{i} \sigma}{v_{m}^{\dagger}}\left(\tilde{x}_{m}-\gamma \tilde{X}_{I}\right) \quad \text { in } d>n_{m}>0,
\end{gathered}
$$

where

$$
\gamma \equiv \rho_{w} / \rho_{m}<1
$$

is the density ratio, and

$$
v_{m}^{\dagger}=v_{m}+\mathrm{i} G_{m} / \rho_{m} \sigma
$$

is the complex viscoelastic parameter for the mud. After using (3.7), the general solutions to (3.12) and (3.13) are, as deduced by $\mathrm{Ng}$ (2000),

$$
\begin{gathered}
\tilde{x}_{w}=\left(1+D \mathrm{e}^{-\lambda_{w} n_{w}}\right) \tilde{X}_{I} \quad \text { in } n_{w}>0, \\
\tilde{x}_{m}=\left(\gamma+E \cosh \lambda_{m} n_{m}+H \sinh \lambda_{m} n_{m}\right) \tilde{X}_{I} \quad \text { in } d>n_{m}>0,
\end{gathered}
$$

where $D, E$ and $H$ are constants,

$$
\lambda_{w}^{2}=-\mathrm{i} \sigma / \nu_{w} \quad \text { or } \quad \lambda_{w}=(1-\mathrm{i}) / \delta_{w},
$$

and

in which

$$
\lambda_{m}^{2}=-\mathrm{i} \sigma / \nu_{m}^{\dagger} \quad \text { or } \quad \lambda_{m}=\left[\lambda_{v}^{-2}-\lambda_{e}^{-2}\right]^{-1 / 2},
$$

$$
\lambda_{v}^{2}=-\mathrm{i} \sigma / v_{m}=(1-\mathrm{i})^{2} / \delta_{m}^{2}, \quad \lambda_{e}^{2}=\rho_{m} \sigma^{2} / G_{m} .
$$

Recall that $\delta_{w, m}=\left(2 v_{w, m} / \sigma\right)^{1 / 2}$ are the respective Stokes boundary-layer thicknesses in the water and mud layers. Further using the boundary conditions (3.4)-(3.6), we may determine the constants as follows:

$$
\begin{gathered}
D=\frac{-\gamma \zeta-(1-\gamma) \zeta \cosh \lambda_{m} d}{\zeta \cosh \lambda_{m} d+\gamma \sinh \lambda_{m} d}, \\
E=-\gamma, \\
H=\frac{\gamma(1-\gamma)+\gamma^{2} \cosh \lambda_{m} d+\gamma \zeta \sinh \lambda_{m} d}{\zeta \cosh \lambda_{m} d+\gamma \sinh \lambda_{m} d},
\end{gathered}
$$

where

$$
\zeta \equiv \lambda_{w} / \lambda_{m}=\left(v_{m}^{\dagger} / v_{w}\right)^{1 / 2}
$$

is a complex parameter equal to the square root of the ratio of the viscoelastic parameter of mud to the viscosity of water. Now, the vertical displacement for each layer can be found from the continuity equation (3.1) as follows:

$$
\begin{gathered}
\tilde{z}_{m}\left(n_{m}\right)=-\mathrm{i} k \int_{0}^{n_{m}} \tilde{x}_{m} \mathrm{~d} n_{m} \quad \text { in } d>n_{m}>0, \\
\tilde{z}_{w}\left(n_{w}\right)=\tilde{z}_{m}(d)-\mathrm{i} k \int_{0}^{n_{w}} \tilde{x}_{w} \mathrm{~d} n_{w} \quad \text { in } n_{w}>0 .
\end{gathered}
$$

On substituting $\tilde{x}_{w}$ and $\tilde{x}_{m}$ into the integrals, the equations above give

$$
\begin{gathered}
\tilde{z}_{m}\left(n_{m}\right)=-\mathrm{i} k \lambda_{m}^{-1}\left[\gamma\left(\lambda_{m} n_{m}-\sinh \lambda_{m} n_{m}\right)+H\left(\cosh \lambda_{m} n_{m}-1\right)\right] \tilde{X}_{I}, \\
\tilde{z}_{w}\left(n_{w}\right)=\tilde{z}_{m}(d)-\mathrm{i}\left[k n_{w}-k D \lambda_{w}^{-1}\left(\mathrm{e}^{-\lambda_{w} n_{w}}-1\right)\right] \tilde{X}_{I} .
\end{gathered}
$$


The dynamic pressure in the mud layer can be deduced from (3.8) and (3.9):

$$
\tilde{p}_{m}(n)=-\mathrm{i} \rho_{w} \sigma^{2} k^{-1} \tilde{X}_{I} .
$$

We have so far found the boundary-layer solutions in terms of $X_{I}$, which is the near-bottom horizontal displacement of the core flow.

We may now adopt from $\mathrm{Ng}(2004 a)$ the most general first-order solution that is valid for the entire water column $(0>\beta>-h)$, including the free-surface and interfacial boundary layers:

$$
\begin{gathered}
\tilde{x}_{w}(\beta)=\left[\cosh k(h+\beta)+B \sinh k(h+\beta)+D \mathrm{e}^{-\lambda_{w}(h+\beta)}+C \mathrm{e}^{\lambda_{w} \beta}\right] \tilde{X}_{I}, \\
\tilde{z}_{w}(\beta)=-\mathrm{i}\left[\sinh k(h+\beta)+B \cosh k(h+\beta)-k \lambda_{w}^{-1}\left(D \mathrm{e}^{-\lambda_{w}(h+\beta)}-C \mathrm{e}^{\lambda_{w} \beta}\right)\right] \tilde{X}_{I},
\end{gathered}
$$

where $D$ is already given by (3.21), and $B$ and $C$ are constants to be determined below. The terms associated with $C$ and $D$ are appreciable only in the free-surface and interfacial boundary layers, respectively. By matching the asymptotic limit of (3.31) as $\beta \rightarrow-h$ with (3.28), $B$ is found to be

$$
\begin{aligned}
B & =\mathrm{i} \tilde{z}_{m}(d) / \tilde{X}_{I}+k D / \lambda_{w} \\
& =k \lambda_{m}^{-1}\left[\gamma\left(\lambda_{m} d-\sinh \lambda_{m} d\right)+H\left(\cosh \lambda_{m} d-1\right)\right]+k \lambda_{w}^{-1} D,
\end{aligned}
$$

or the interfacial wave amplitude can be expressed in terms of $B$ :

$$
b=\tilde{z}_{m}(d)=-\mathrm{i}\left(B-k \lambda_{w}^{-1} D\right) \tilde{X}_{I} .
$$

One can readily check that, normally, $B=O\left(\varepsilon_{m}\right)$ and $b=O\left(\varepsilon_{m} a\right)$, confirming that the interfacial wave amplitude is an order of magnitude smaller than the surface wave amplitude. The constant $C$ can be found by imposing the condition of zero shear stress on the free surface:

$$
\frac{\partial \dot{x}_{w 1}}{\partial \beta}+\frac{\partial \dot{z}_{w 1}}{\partial \alpha}=0 \quad \text { on } \beta=0,
$$

which on substituting (3.30) and (3.31) gives

$$
C=-2 k \lambda_{w}^{-1}[\sinh k h+B \cosh k h]+O\left(\varepsilon_{w}\right)^{2} .
$$

Using the kinematic free-surface condition that $\tilde{z}_{w}=a$ on $\beta=0$, we may obtain an expression for $\tilde{X}_{I}$ :

$$
\tilde{X}_{I}=\frac{\mathrm{i} a}{\sinh k h+B \cosh k h}+O\left(\varepsilon_{w}\right)^{2} .
$$

Further using the condition of zero normal stress on the free surface $(\mathrm{Ng} 2004 a)$ :

$$
\begin{aligned}
& -p_{w 1}+2 \mu_{w} \frac{\partial \dot{z}_{w 1}}{\partial \beta}=0 \\
\Rightarrow & g \tilde{z}_{w}+\sigma^{2}\left(k \lambda_{w}\right)^{-2}\left[\tilde{z}_{w}^{\prime \prime \prime}-\left(2 k^{2}+\lambda_{w}^{2}\right) \tilde{z}_{w}^{\prime}\right]=0 \quad \text { on } \beta=0,
\end{aligned}
$$

we may obtain an eigenvalue relation for $k$ :

$$
\frac{\sigma^{2}}{g k}=\frac{\tanh k h+B}{1+B \tanh k h}+O\left(\varepsilon_{w}\right)^{2} .
$$

Since $B=O\left(\varepsilon_{m}\right)$, we may expand the wavenumber into $k=k_{1}+k_{2}+\cdots$, where $k_{i}=O\left(\varepsilon_{m}\right)^{i-1}$. While the leading-order wavenumber is real and determined by the familiar dispersion relation

$$
\sigma^{2}=g k_{1} \tanh k_{1} h,
$$


the next order $k_{2}$ is complex and given by

$$
k_{2}=-\frac{B k_{1}}{\sinh k_{1} h \cosh k_{1} h+k_{1} h} .
$$

The imaginary part of $k_{2}$ corresponds to the rate of spatial attenuation of the wave:

$$
D_{m / w} \equiv \operatorname{Im}\left(k_{2}\right)=-\frac{k_{1} \operatorname{Im}(B)}{\sinh k_{1} h \cosh k_{1} h+k_{1} h}=-\frac{2 k_{1} \operatorname{Im}(B)}{\sinh 2 k_{1} h+2 k_{1} h},
$$

where Im stands for the imaginary part. In the limiting case of clear water only (i.e. $d=0$ ), the classical wave attenuation rate may be recovered:

$$
D_{w}=\frac{\delta_{w} k_{1}^{2}}{\sinh 2 k_{1} h+2 k_{1} h} .
$$

The effects of mud viscoelasticity on the transformation of the surface and interfacial waves, including wave attenuation, have been examined by Zhang \& $\mathrm{Ng}(2006 a)$.

At this point, we have solved the first-order problem for the oscillatory particle displacements in the mud and water layers, and also for the surface and interfacial wave characteristics. The Newtonian limit (i.e. $G_{m}=0$ ) of the present boundary-layer model, as presented by $\mathrm{Ng}(2000)$, has been tested by comparing results on the wave damping factor for an example case of deep water as considered by Dalrymple \& Liu (1978), who solved the problem using a complete model theory. In the example case, $h=4 \mathrm{~m}, \sigma=1.2566 \mathrm{~s}^{-1}, \gamma=0.57, \varepsilon_{m}=0.09$ and $\varepsilon_{w}=4.6 \times 10^{-4}$. The comparison in this example reveals that the boundary-layer model can agree very well with the complete model as long as the mud depth is not thicker than $30 \%$ of the water depth.

Let us now proceed to the second order to determine the steady drift or mass transport velocity in the water layer. Since our interest is in a horizontal length scale comparable to the wavelength, the higher-order wave-attenuation effects can be ignored. From here on, we shall not distinguish $k_{1}$ from $k$, which is simply taken as a real quantity.

\section{Second-order solutions}

As was remarked by MacPherson (1980), the mechanical representation of the Voigt model is a spring and a dashpot operating in parallel. When subjected to a steady load, the system will initially deform at a rate controlled by the damping of the dashpot, but the deformation will ultimately approach a finite value depending on the elasticity of the spring, as the dashpot gradually loses its effect. Zhang \& Ng (2006b) studied the mass transport due to wave forcing undergone by a single layer of viscoelastic mud modelled as a Voigt body. They confirmed that a steady mass transport velocity cannot be sustained in such a medium. The second-order time-mean motion will instead die out exponentially over a time scale equal to the ratio of the viscosity to the shear modulus, $\mu_{m} / G_{m}$. Therefore, for a mud with sufficient strength such that $G_{m} / \mu_{m} \geqslant O(\sigma)$, the time-mean motion of the mud is short lived, as the ultimate mean deformation can be attained readily within several wave periods or so. Here, we have assumed that $G_{m} / \mu_{m}=O(1) \mathrm{s}^{-1}$, and therefore the condition can be met as long as $\sigma \leqslant O(1) \mathrm{s}^{-1}$ (i.e. the wave period is $6 \mathrm{~s}$ or longer). The period of transient mean motion will then be of no appreciable significance and we may consider an instant of time when the mud has already stopped undergoing mean motion and attained the equilibrium steady deformation. As a result, the present problem is reduced to finding only the mass transport taking place in the water 
layer, which is influenced by the mud viscoelasticity through the first-order oscillatory motion of the water itself.

In the absence of mean motion of the mud, the mass transport problem can be handled as if the system consisted only of clear water. The analysis therefore resembles that for mass transport in a single layer of water, as has been presented by Ünlüata \& Mei (1970) and $\mathrm{Ng}(2004 a)$.

The mass transport velocity, which is the steady component of the second-order Lagrangian velocity, is purely horizontal when induced by a progressive surface wave. Let us denote it by

$$
u_{L}(\beta) \equiv \overline{\dot{x}}_{w 2},
$$

where the overbar denotes time average over one wave period. This mass transport velocity is governed by the time-averaged second-order horizontal momentum equation:

$$
\frac{\partial}{\partial \alpha}\left(\frac{\bar{p}_{w 2}}{\rho}+g \bar{z}_{w 2}\right)-v_{w} \frac{\mathrm{d}^{2} u_{L}}{\mathrm{~d} \beta^{2}}=\bar{X}_{w 2}
$$

where

$$
\bar{X}_{w 2}=v_{w} \sigma k\left[\frac{1}{2} k^{2}\left|\tilde{z}_{w}\right|^{2}-2 \operatorname{Re}\left(\tilde{x}_{w}^{*} \tilde{x}_{w}^{\prime \prime}\right)-k \operatorname{Im}\left(\tilde{z}_{w}^{*} \tilde{x}_{w}^{\prime}\right)-\frac{3}{2}\left|\tilde{x}_{w}^{\prime}\right|^{2}\right]
$$

in which the asterisk denotes complex conjugate. From the second-order vertical momentum equation, we may obtain, after taking time averages,

$$
\frac{\bar{p}_{w 2}}{\rho}+g \bar{z}_{w 2}=g \bar{\eta}_{2}+\int_{0}^{\beta} \bar{Z}_{w 2} \mathrm{~d} \beta,
$$

where $\bar{\eta}_{2}(\alpha)$ is the set-up or mean displacement of the free surface, and $\bar{Z}_{w 2}$ is a function of $\beta$ only. Therefore, substituting (4.4) into (4.2), we obtain

$$
v_{w} \frac{\mathrm{d}^{2} u_{L}}{\mathrm{~d} \beta^{2}}=g \frac{\mathrm{d} \bar{\eta}_{2}}{\mathrm{~d} \alpha}-\bar{X}_{w 2},
$$

where, as will be determined in (4.12), the spatial gradient of the free surface set-up, $\mathrm{d} \bar{\eta}_{2} / \mathrm{d} \alpha$, corresponds to a counter-balancing flux when the domain is closed; this term is nevertheless subdominant in the boundary layers.

Following Ünlüata \& Mei (1970) and $\mathrm{Ng}$ (2004a), we may derive from (4.5) the mass transport velocity in the entire water layer as follows. First, in the bottom boundary layer $(\beta \sim-h)$,

$$
u_{L}=\sigma k\left|\tilde{X}_{I}\right|^{2}\left\{2 \operatorname{Re}\left[D\left(\mathrm{e}^{-\lambda_{w}(h+\beta)}-1\right)\right]+\frac{3}{4}|D|^{2}\left(\mathrm{e}^{-2(h+\beta) / \delta_{w}}-1\right)\right\},
$$

which tends to the following limit at the outer edge of the boundary layer:

$$
\left.u_{L}\right|_{h+\beta \gg \delta_{w}}=-\sigma k\left|\tilde{X}_{I}\right|^{2}\left[2 \operatorname{Re}(D)+\frac{3}{4}|D|^{2}\right] .
$$

Secondly, in the free-surface boundary layer $(\beta \sim 0)$,

$$
\frac{\mathrm{d} u_{L}}{\mathrm{~d} \beta}=2 \sigma k\left|\tilde{X}_{I}\right|^{2} \operatorname{Re}\left[\lambda_{w} C\left(\cosh k h+B^{*} \sinh k h\right)\left(\mathrm{e}^{\lambda_{w} \beta}-1\right)\right],
$$

which tends to the following limit at the outer edge of the boundary layer

$$
\left.\frac{\mathrm{d} u_{L}}{\mathrm{~d} \beta}\right|_{-\beta \gg \delta_{w}}=2 \sigma k^{2}\left|\tilde{X}_{I}\right|^{2}\left[\left(1+|B|^{2}\right) \sinh 2 k h+2 \operatorname{Re}(B) \cosh 2 k h\right] .
$$

Finally, in the water core region that is far away from the bottom and free-surface 
boundary layers,

$$
\begin{aligned}
u_{L}(\beta)= & \frac{g}{2 v_{w}} \frac{\mathrm{d} \bar{\eta}_{2}}{\mathrm{~d} \alpha}\left(\beta^{2}-h^{2}\right)+\frac{1}{2} \sigma k\left|\tilde{X}_{I}\right|^{2}\left\{\left(1+|B|^{2}\right)[\cosh 2 k(h+\beta)\right. \\
& -1+2 k(h+\beta) \sinh 2 k h]+2 \operatorname{Re}(B)[\sinh 2 k(h+\beta) \\
& \left.+2 k(h+\beta) \cosh 2 k h]-4 \operatorname{Re}(D)-\frac{3}{2}|D|^{2}\right\} .
\end{aligned}
$$

The surface set-up gradient is zero when the domain is unbounded, but is to be associated with a return current when the domain is closed. The return current is to balance the forward flux so that the net discharge is zero. This can be worked out as follows. The total flux due to the mass transport is

$$
\begin{aligned}
Q= & \int_{-h}^{0} u_{L} \mathrm{~d} \beta \\
= & -\frac{g h^{3}}{3 v_{w}} \frac{\mathrm{d} \bar{\eta}_{2}}{\mathrm{~d} \alpha}+\frac{1}{2} \sigma k h\left|\tilde{X}_{I}\right|^{2}\left[\left(1+|B|^{2}\right)\left(\frac{\sinh 2 k h}{2 k h}-1+k h \sinh 2 k h\right)\right. \\
& \left.+2 \operatorname{Re}(B)\left(\frac{\cosh 2 k h-1}{2 k h}+k h \cosh 2 k h\right)-4 \operatorname{Re}(D)-\frac{3}{2}|D|^{2}\right],
\end{aligned}
$$

which is equal to zero when the set-up gradient is given by

$$
\begin{aligned}
\frac{g h^{2}}{2 v_{w}} \frac{\mathrm{d} \bar{\eta}_{2}}{\mathrm{~d} \alpha}= & \frac{3}{4} \sigma k\left|\tilde{X}_{I}\right|^{2}\left[\left(1+|B|^{2}\right)\left(\frac{\sinh 2 k h}{2 k h}-1+k h \sinh 2 k h\right)\right. \\
& \left.+2 \operatorname{Re}(B)\left(\frac{\cosh 2 k h-1}{2 k h}+k h \cosh 2 k h\right)-4 \operatorname{Re}(D)-\frac{3}{2}|D|^{2}\right] .
\end{aligned}
$$

On substituting this back into (4.10), we may obtain an expression for the mass transport velocity encompassing the two possible far end conditions for the domain:

$$
\begin{aligned}
u_{L}(\beta)= & \frac{1}{2} \sigma k\left|\tilde{X}_{I}\right|^{2}\left\{\left(1+|B|^{2}\right)[\cosh 2 k(h+\beta)-1+2 k(h+\beta) \sinh 2 k h]\right. \\
& +2 \operatorname{Re}(B)[\sinh 2 k(h+\beta)+2 k(h+\beta) \cosh 2 k h]-4 \operatorname{Re}(D)-\frac{3}{2}|D|^{2} \\
& +\frac{3 Y}{2}\left(\frac{\beta^{2}}{h^{2}}-1\right)\left[\left(1+|B|^{2}\right)\left(\frac{\sinh 2 k h}{2 k h}-1+k h \sinh 2 k h\right)\right. \\
& \left.\left.+2 \operatorname{Re}(B)\left(\frac{\cosh 2 k h-1}{2 k h}+k h \cosh 2 k h\right)-4 \operatorname{Re}(D)-\frac{3}{2}|D|^{2}\right]\right\},
\end{aligned}
$$

where $Y$ is an integer parameter switching on or off the closed-domain condition:

$$
Y= \begin{cases}0 & \text { if domain is open ended, } \\ 1 & \text { if domain is bounded. }\end{cases}
$$

Let us check the limiting case when the mud layer does not exist, or $d=0$, to which the following relationships apply: (i) in the absence of resonance, all terms associated with $B$ are always subdominant and can be ignored, (ii) $D=-1$, and (iii) $\tilde{X}_{I} \approx i a / \sinh k h$. By virtue of these limiting relationships, we readily find that (4.13) reduces to

$$
\begin{aligned}
u_{L}(\beta)= & \frac{\sigma k a^{2}}{4 \sinh ^{2} k h}[3+2 \cosh 2 k(h+\beta)+4 k(h+\beta) \sinh 2 k h \\
& \left.+3 Y\left(\frac{\beta^{2}}{h^{2}}-1\right)\left(\frac{3}{2}+k h \sinh 2 k h+\frac{\sinh 2 k h}{2 k h}\right)\right] \quad \text { for } d=0,
\end{aligned}
$$


which accords with the one deduced by Ünlüata \& Mei (1970) for mass transport due to progressive waves in a one-layer system. The expression (4.13) is for a two-layer system, where the mass transport in the upper-layer fluid is affected, through the parameters $B$ and $D$, by the viscoelasticity of the lower-layer material. The mass transport is purely horizontal under a progressive wave, and the expressions deduced above are hence good, even when the surface-wave amplitude is much larger than the Stokes boundary-layer thickness in water (e.g. Wen \& Liu 1995a).

\section{Numerical results and discussions}

In this section, we will use numerical examples to examine the effects of viscoelasticity on the mass transport in the water layer. Let us first introduce the following normalized variables, which are distinguished by an overhead caret:

$$
\left.\begin{array}{l}
\left(\hat{u}_{w 1}, \hat{v}_{w 1}\right)=\left(\dot{x}_{w 1}, \dot{z}_{w 1}\right) /\left|\dot{X}_{I}\right|, \quad \hat{u}_{L}=u_{L} / \varepsilon \sigma a, \quad \hat{t}=\sigma t / \pi, \\
\hat{Q}=Q / \sigma a^{2}, \quad \hat{k}=k h, \quad \hat{\beta}=\beta / h, \quad \hat{b}=b / \varepsilon \delta_{w}, \\
\left(\hat{\lambda}_{m}, \hat{\lambda}_{v}, \hat{\lambda}_{e}\right)=\delta_{w}\left(\lambda_{m}, \lambda_{v}, \lambda_{e}\right), \quad\left(\hat{n}_{w}, \hat{d}, \hat{\delta}_{m}\right)=\left(n_{w}, d, \delta_{m}\right) / \delta_{w} .
\end{array}\right\}
$$

Note that $\delta_{w}$ has been chosen as the normalization scale even for the mud layer, since it is desirable to leave $\delta_{m}$ as a free parameter. By this normalization, we may further express

$$
\begin{gathered}
\hat{\lambda}_{m}=\left(\hat{\lambda}_{v}^{-2}-\hat{\lambda}_{e}^{-2}\right)^{-1 / 2}, \\
\hat{\lambda}_{v}=(1-\mathrm{i}) / \hat{\delta}_{m}, \quad \hat{\lambda}_{e}=\delta_{w}\left(\rho_{m} \sigma^{2} / G_{m}\right)^{1 / 2}=\left(2 \mu_{w} \sigma / \gamma G_{m}\right)^{1 / 2},
\end{gathered}
$$

and

$$
\zeta=(1-\mathrm{i}) / \hat{\lambda}_{m}
$$

In terms of the normalized variables and parameters, we may express the quantities of interest as follows. The normalized interfacial wave amplitude is

$$
|\hat{b}|=\left|\frac{\gamma\left(\hat{\lambda}_{m} \hat{d}-\sinh \hat{\lambda}_{m} \hat{d}\right)+H\left(\cosh \hat{\lambda}_{m} \hat{d}-1\right)}{\hat{\lambda}_{m}(\sinh \hat{k}+B \cosh \hat{k})}\right| .
$$

The mass transport velocity in the bottom boundary layer as given by (4.6) is

$$
\hat{u}_{L}=|\sinh \hat{k}+B \cosh \hat{k}|^{-2}\left\{2 \operatorname{Re}\left[D\left(\mathrm{e}^{-(1-\mathrm{i}) \hat{n}_{w}}-1\right)\right]+\frac{3}{4}|D|^{2}\left(\mathrm{e}^{-2 \hat{n}_{w}}-1\right)\right\} .
$$

The mass transport velocity in the core region as given by (4.13) is

$$
\begin{aligned}
\hat{u}_{L}(\hat{\beta})= & \frac{1}{2}|\sinh \hat{k}+B \cosh \hat{k}|^{-2}\left\{\left(1+|B|^{2}\right)[\cosh 2 \hat{k}(1+\hat{\beta})-1+2 \hat{k}(1+\hat{\beta}) \sinh 2 \hat{k}]\right. \\
& +2 \operatorname{Re}(B)[\sinh 2 \hat{k}(1+\hat{\beta})+2 \hat{k}(1+\hat{\beta}) \cosh 2 \hat{k}]-4 \operatorname{Re}(D)-\frac{3}{2}|D|^{2} \\
& +\frac{3 Y}{2}\left(\hat{\beta}^{2}-1\right)\left[\left(1+|B|^{2}\right)\left(\frac{\sinh 2 \hat{k}}{2 \hat{k}}-1+\hat{k} \sinh 2 \hat{k}\right)\right. \\
& \left.\left.+2 \operatorname{Re}(B)\left(\frac{\cosh 2 \hat{k}-1}{2 \hat{k}}+\hat{k} \cosh 2 \hat{k}\right)-4 \operatorname{Re}(D)-\frac{3}{2}|D|^{2}\right]\right\}
\end{aligned}
$$


and the total flux for an open-ended domain as given by (4.11) is

$$
\begin{aligned}
\hat{Q}= & \frac{\hat{k}}{2}|\sinh \hat{k}+B \cosh \hat{k}|^{-2}\left[\left(1+|B|^{2}\right)\left(\frac{\sinh 2 \hat{k}}{2 \hat{k}}-1+\hat{k} \sinh 2 \hat{k}\right)\right. \\
& \left.+2 \operatorname{Re}(B)\left(\frac{\cosh 2 \hat{k}-1}{2 \hat{k}}+\hat{k} \cosh 2 \hat{k}\right)-4 \operatorname{Re}(D)-\frac{3}{2}|D|^{2}\right] .
\end{aligned}
$$

In the expressions above, the parameters $B, D$ and $H$ are as follows:

$$
\begin{gathered}
B=\varepsilon_{w}\left\{\hat{\lambda}_{m}^{-1}\left[\gamma\left(\hat{\lambda}_{m} \hat{d}-\sinh \hat{\lambda}_{m} \hat{d}\right)+H\left(\cosh \hat{\lambda}_{m} \hat{d}-1\right)\right]+\frac{(1+\mathrm{i})}{2} D\right\}, \\
D=\frac{-\gamma \zeta-(1-\gamma) \zeta \cosh \hat{\lambda}_{m} \hat{d}}{\zeta \cosh \hat{\lambda}_{m} \hat{d}+\gamma \sinh \hat{\lambda}_{m} \hat{d}},
\end{gathered}
$$

and

$$
H=\frac{\gamma(1-\gamma)+\gamma^{2} \cosh \hat{\lambda}_{m} \hat{d}+\gamma \zeta \sinh \hat{\lambda}_{m} \hat{d}}{\zeta \cosh \hat{\lambda}_{m} \hat{d}+\gamma \sinh \hat{\lambda}_{m} \hat{d}} .
$$

To evaluate the quantities above, we must specify six basic input parameters: (i) $\gamma$ for the density ratio, (ii) $\hat{\lambda}_{e}$ for the elasticity of the mud, (iii) $\hat{\delta}_{m}$ for the viscosity of the mud, (iv) $\hat{d}$ for the mud depth, (v) $\hat{k}$ for the ratio of the water depth to the wavelength, and (vi) $\varepsilon_{w}$ for the ratio of the Stokes boundary-layer thickness in water to the wavelength. To be consistent with the assumptions stated earlier, these parameter should be so chosen that $\left|\hat{\lambda}_{m} \hat{d}\right|=O(1), \hat{k}=O(1)$ and $\varepsilon_{w} \ll 1$. Also, for the mean motion of the mud to die out within a relatively short period of time, we require $\left(\hat{\lambda}_{e} \hat{\delta}_{m}\right)^{2} \leqslant O(1)$.

Recall that we have been considering a partially fluidized mud or a weak soil with a bulk density $1.2-1.3$ times that of clear water, and with the lowest measurable viscoelastic coefficients, i.e. both the viscosity, $\mu_{m}$ (Pas), and the shear modulus of elasticity, $G_{m}(\mathrm{~Pa})$, are $O(10)$. Based on these physical estimates, we choose in our numerical calculations the following input values: (i) $\gamma=0.8$, corresponding to a ratio of $\rho_{m} / \rho_{w}=1.25$; (ii) $\hat{\delta}_{m}=8$, corresponding to $\mu_{m} / \mu_{w}=O\left(10^{2}\right)$ or $\mu_{m}=O(10) \mathrm{Pas}$ if the eddy viscosity of water $v_{w}=O(1) \mathrm{cm}^{2} \mathrm{~s}^{-1}$; and (iii) $\hat{\lambda}_{e}=$ $0.3,0.15,0.1$, corresponding to $G_{m}=O(10) \mathrm{Pa}$, as can be estimated from (5.3). It follows that $\hat{\lambda}_{m}=O(0.1)$, which allows us to consider a mud depth as thick as $\hat{d}=O(10)$, corresponding to a physical mud depth of $d=O(10) \mathrm{cm}$, which accords with the field observation (e.g. Kirby, Hobbs \& Mehta 1989). Finally, since the wavelength is typically several orders of magnitude longer than the Stokes boundarylayer thickness in water, $\varepsilon_{w}=10^{-3}$ is used in our computations.

Let us, for the sake of subsequent discussions, consider two mathematical limits of the present theory. The first limit is when the mud is purely elastic or its viscosity is zero: $v_{m}=0$. In such a limiting case, $\hat{\delta}_{m}=0$ implying $\hat{\lambda}_{m}=\mathrm{i} \hat{\lambda}_{e}$. It follows that, since $|\zeta| \gg 1$, the denominators of the two parameters $D$ and $H$, as given by (5.10) and (5.11), are both approximately equal to $\cos \hat{\lambda}_{e} \hat{d}$. Therefore, the two parameters will blow up when $\hat{\lambda}_{e} \hat{d}=\pi / 2,3 \pi / 2,5 \pi / 2, \ldots$, corresponding to the occurrence of resonance. We have numerically confirmed that, in the case of a pure elastic mud, the interfacial wave amplitude $|\hat{b}|$ will indeed shoot up abruptly at specific values of the mud depth $\hat{d}_{R}=\pi / 2 \hat{\lambda}_{e}, 3 \pi / 2 \hat{\lambda}_{e}, \ldots$ The resonant response is sharper and higher for smaller $\hat{\lambda}_{e}$ (i.e. stronger elasticity), when the resonance occurs to a thicker 

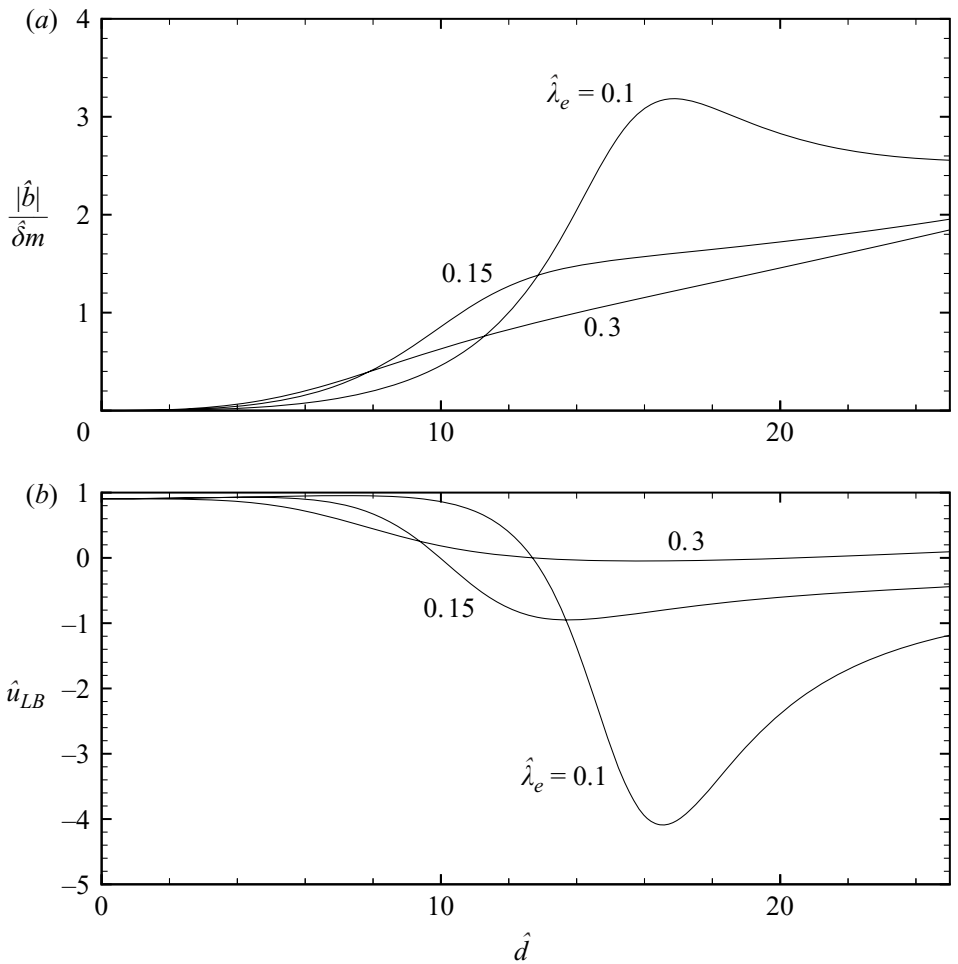

FIGURE 2. Interfacial wave amplitude $|\hat{b}| / \hat{\delta}_{m}$, and mass transport velocity at the outer edge of the bottom boundary layer $\hat{u}_{L B}$, as functions of $\hat{d}$ and $\hat{\lambda}_{e}$.

mud layer. The presence of water overlying the mud $(\gamma \neq 0)$ will keep the resonant amplitude from being blown up to infinity. Nevertheless, the oscillatory motion of mud can be amplified significantly when resonance occurs. We caution that, however, the resonance can only be treated as a singular solution to the present theory if it gives rise to an excessively large amplification, as this may grossly violate the original assumption of small particle displacements and linear elastic response. The present theory will break down when the mud begins to yield plastically.

Practically, fluidized mud is not purely elastic, but has fairly large viscosity that will substantially diminish the resonance amplification. This viscoelastic property of the mud allows us to examine the effects of resonance within the range of validity of the present theory. Figure 2 shows that even when the mud viscosity is as large as $\hat{\delta}_{m}=8$, the resonance can still be appreciable for sufficiently strong elasticity of the mud, say $\hat{\lambda}_{e}<0.15$. We have found that for weaker elasticity such that $\hat{\lambda}_{e} \geqslant 0.3$, resonance is completely suppressed and the interfacial wave amplitude $|\hat{b}|$ increases monotonically

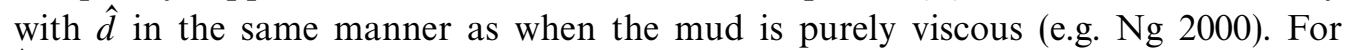
$\hat{\lambda}_{e} \leqslant 0.15$, resonance manifests itself as the interfacial wave amplitude exhibits a local peak at values of $\hat{d}$ close to $\hat{d}_{R}$ given above (e.g. a local peak occurs at $\hat{d}_{R} \approx 16$ for $\left.\hat{\lambda}_{e}=0.1\right)$. The damped resonance gives rise to only an order of unity amplification, and therefore it is legitimate for us to look into its effect on the mass transport velocity.

The second mathematical limit, which has significant implications, is when the parameter $D$ in (3.16) vanishes identically. The first implication is, as is obvious in (3.30) and (3.31), that the boundary layer above the water-mud interface will 
disappear completely. The second implication is, as is obvious in (4.6), that the nearbottom mass transport velocity will vanish too. From (5.10), $D=0$ happens when $\cosh \hat{\lambda}_{m} \hat{d}=-\gamma /(1-\gamma)$. Since the fluidized mud is practically no more than $30 \%$ denser than clear water, the density ratio $\gamma$ can be limited to the range $0.5<\gamma<1$, for which the equation above has no roots when $\hat{\lambda}_{m}$ is real or imaginary. In other words, $D=0$ will not happen when the mud is purely viscous or elastic. The condition can be satisfied, however, when the mud is viscoelastic such that $\hat{\lambda}_{m}$ has non-zero real $\left(\hat{\lambda}_{m r}\right)$ and imaginary $\left(\hat{\lambda}_{m i}\right)$ parts. On substituting $\hat{\lambda}_{m}=\hat{\lambda}_{m r}+i \hat{\lambda}_{m i}$, the equation above can be written as

$$
\cosh \hat{\lambda}_{m r} \hat{d} \cos \hat{\lambda}_{m i} \hat{d}+\mathrm{i} \sinh \hat{\lambda}_{m r} \hat{d} \sin \hat{\lambda}_{m i} \hat{d}=-\gamma /(1-\gamma) .
$$

Obviously, this equation can be satisfied when

$$
\hat{\lambda}_{m r} \hat{d}= \pm \cosh ^{-1}\left(\frac{\gamma}{1-\gamma}\right)
$$

and

$$
\hat{\lambda}_{m i} \hat{d}= \pm \pi, \pm 3 \pi, \pm 5 \pi, \ldots,
$$

so that $\cos \hat{\lambda}_{m i} \hat{d}=-1$ and $\sin \hat{\lambda}_{m i} \hat{d}=0$. By (5.2) and (5.3), we may express

$$
\hat{\lambda}_{m}=\hat{\lambda}_{e}\left[\frac{-1-\mathrm{i} \hat{\lambda}_{e}^{2} \hat{\delta}_{m}^{2} / 2}{1+\left(\hat{\lambda}_{e}^{2} \hat{\delta}_{m}^{2} / 2\right)^{2}}\right]^{1 / 2},
$$

from which $\hat{\lambda}_{m r}$ and $\hat{\lambda}_{m i}$ can be obtained readily. After some algebra, the two conditions (5.13) and (5.14) can be shown to be equivalent to

$$
\hat{\lambda}_{e}^{2} \hat{\delta}_{m}^{2}=2\left(S_{j}^{2}-1\right)^{1 / 2}
$$

and

$$
\hat{\lambda}_{e}^{2} \hat{d}^{2}=2\left(S_{j}-1\right)^{-1}\left[S_{j} \cosh ^{-1}\left(\frac{\gamma}{1-\gamma}\right)\right]^{2}
$$

where

$$
\begin{gathered}
S_{j}=\frac{1+\xi_{j}}{1-\xi_{j}}, \\
\xi_{j}=\left[(j \pi)^{-1} \cosh ^{-1}\left(\frac{\gamma}{1-\gamma}\right)\right]^{2} \quad(j=1,3,5, \ldots) .
\end{gathered}
$$

For given $\gamma$ and one of the three parameters $\hat{d}, \hat{\delta}_{m}$ and $\hat{\lambda}_{e}$, (5.16) and (5.17) can be used to determine the other two parameters corresponding to $D=0$. As in the present example, when $\gamma=0.8$ and $\hat{\delta}_{m}=8$, the first solutions given by the two equations are $\hat{\lambda}_{e}=0.27$ and $\hat{d}=22.2$. The parameter $D$ vanishes when this set of values is met, implying that $x_{w 1}\left(n_{w}=0\right)=X_{I}$ or the interface will oscillate in exactly the same phase with the same amplitude as the near-bottom water particles; this will eliminate the need for a boundary layer to exist above the interface. This is a distinct phenomenon that can happen only to a viscoelastic mud. The disappearance of the interfacial boundary layer will cause the mass transport velocity to vanish there as well. A slight departure from $D=0$ will turn the real part of $D$ to either positive or negative, which according to (4.7) (where $|D|^{2}$ is negligible for a small departure from zero) will 
make the near-bottom mass transport velocity negative or positive, respectively. The possibility of zero or negative mass transport velocity in a progressive wave boundary layer (which will not happen when the bottom material is purely viscous) is indeed remarkable and deserves further examination. Of course, for the near-bottom mass transport velocity to vanish, $D=0$ is only a sufficient, but not necessary condition; the more general condition is, according to $(4.7), \operatorname{Re}(D)=-3|D|^{2} / 8$.

Let us revisit figure 2, which shows also the mass transport velocity at the outer edge of the bottom boundary layer $\hat{u}_{L B} \equiv \hat{u}_{L}(\hat{\beta}=-1)$ as a function of mud depth $\hat{d}$ and elasticity $\hat{\lambda}_{e}$. First note that for a rigid bed (i.e. in the absence of the mud layer, $\hat{d}=0)$, the near-bed mass transport velocity $\hat{u}_{L B}=(5 / 4) \sinh ^{2} \hat{k}=0.905$ for $\hat{k}=1$. As was noted by $\mathrm{Ng}(2000)$, the existence of a layer of viscous mud is to decrease $\hat{u}_{L B}$; the larger the mud depth $\hat{d}$, the smaller $\hat{u}_{L B}$. Figure 2 suggests that such a trait is also exhibited by the viscosity-dominated case of $\hat{\lambda}_{e}=0.3$, in which $\hat{u}_{L B}$ decreases monotonically with increasing $\hat{d}$. By contrast, the cases with stronger elasticity $\hat{\lambda}_{e}=0.15,0.1$ exhibit qualitatively different dependence of $\hat{u}_{L B}$ on $\hat{d}$. When the mud is not deep enough for resonance to occur, an elasticity-dominated mud layer can lead to an increased $\hat{u}_{L B}$. Similar findings have been reported by Wen \& Liu (1995a), who found that, for an infinitely deep elastic bed, the near-bed mass transport velocity can be enhanced by as much as twice that for a rigid bed. We here consider a very thin mud layer, and therefore the enhancement of the near-bed mass transport velocity is relatively negligible (e.g. for $\hat{\lambda}_{e}=0.1, \hat{u}_{L B}$ is only increased to a maximum value of 0.953 when $\hat{d}=0.7)$. More dramatic and reversed effects are seen, however, when the resonance mud depth is approached: the near-bed mass transport velocity $\hat{u}_{L B}$ drops significantly to cross the value of zero to reach a minimum that is negative and can be several times larger in magnitude than that for a rigid bed. It is clear that a larger magnitude of the local minimum $\hat{u}_{L B}$ is associated with a stronger resonant response resulting from a larger value of the elastic modulus of the mud layer.

That an elasticity-induced resonance amplification of the mud motion can lead to reversed mass transport of water in the interfacial boundary layer can be understood with the aid of figure 3 . This figure serves to provide a comparison of the boundarylayer profiles of the amplitude of the first-order horizontal velocity $\left|\hat{u}_{w 1}\right|\left(\hat{n}_{w}\right)$ for three cases: $(a)$ a resonance case with negative mass transport, $\hat{d}=16$ and $\hat{\lambda}_{e}=0.1 ;(b)$ a case with practically zero mass transport, $\hat{d}=16$ and $\hat{\lambda}_{e}=0.3 ;(c)$ positive mass transport in the limiting case of a rigid bottom, $\hat{d}=0$. From the correlation between the horizontal and vertical components of the first-order velocity at $\hat{n}_{w}=1$ (shown in the left-hand column of the figure), it can be inferred that in each case a water particle will follow a clockwise closed trajectory in one wave period at the first order. In other words, a particle will be at a slightly higher elevation when it moves forward than when it moves backward. A net particle drift of second order will result if there is a velocity differential with height. The drift will be positive/negative if the velocity amplitude increases/decreases with height. In case $(a)$, the resonance amplification causes the interface to oscillate with a larger horizontal amplitude than that at the outer edge of the boundary layer: $\left|x_{w 1}\right|\left(\hat{n}_{w}=0\right)>\left|X_{I}\right|$. As a result, the velocity amplitude decreases with height, and the mass transport velocity is negative. In case $(b), x_{w 1}\left(\hat{n}_{w}=0\right) \approx X_{I}$ in terms of both phase and amplitude, and the velocity is almost uniform across the boundary layer. This is a case in which the boundary layer virtually disappears. In the absence of an appreciable velocity gradient, the mass transport is nearly zero in this case. In the limiting case $(c)$, the mud is absent and the water bottom is stationary. The velocity amplitude, which is zero on the bottom, then increases with height, and the mass transport velocity is positive, as is well known in the literature. 

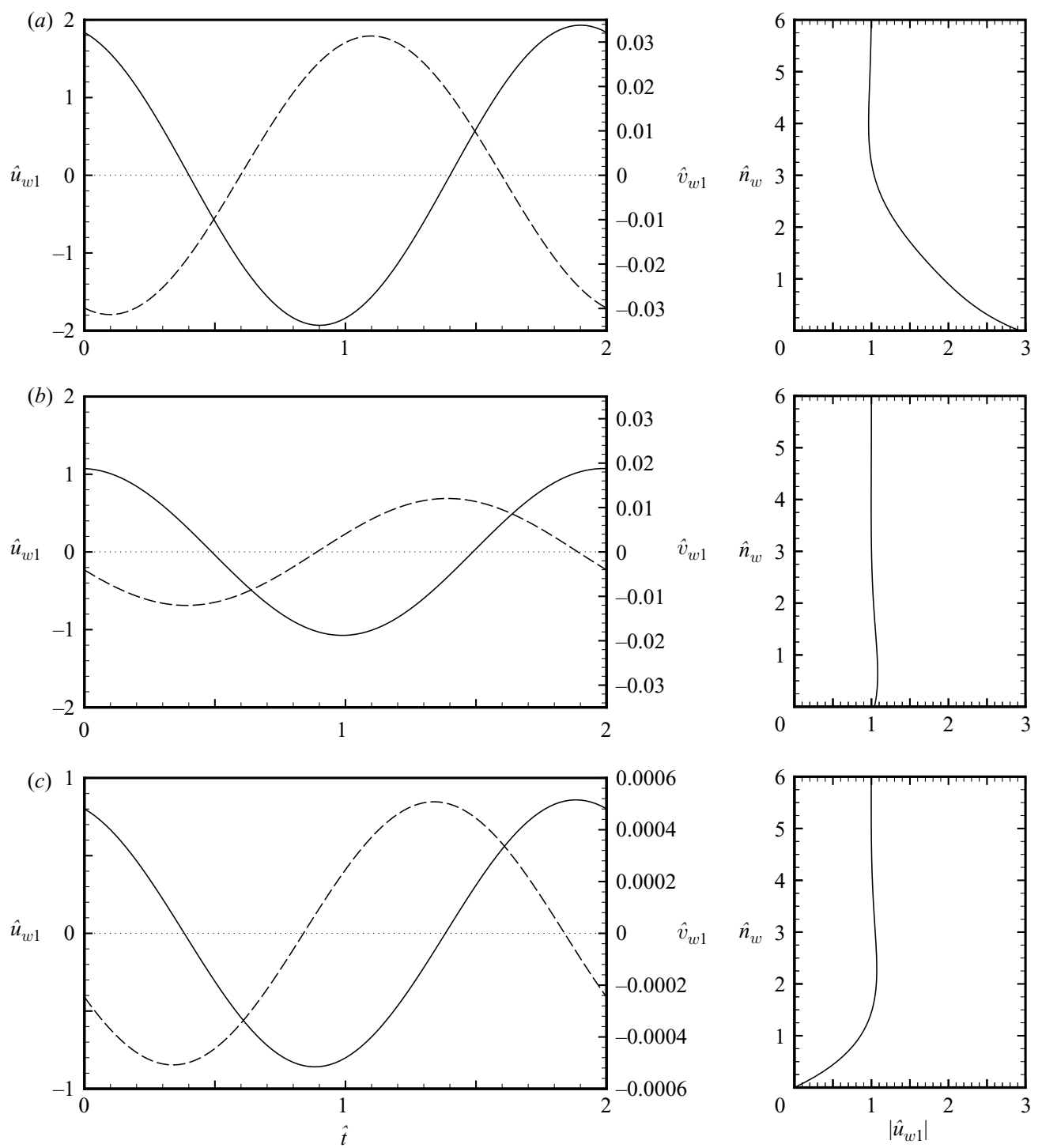

FIGURE 3. Horizontal (solid) and vertical (dashes) components of first-order velocity $\left(\hat{u}_{w 1}, \hat{v}_{w 1}\right)$ at $\hat{n}_{w}=1$ as functions of $\hat{t}$ (left-hand column); amplitude of first-order horizontal velocity $\left|\hat{u}_{w 1}\right|$ as a function of $\hat{n}_{w}$ (right-hand column), where $(a) \hat{d}=16$ and $\hat{\lambda}_{e}=0.1,(b) \hat{d}=16$ and $\hat{\lambda}_{e}=0.3$, (c) $\hat{d}=0$.

Figure 4 shows the mass transport velocity profiles $\hat{u}_{L}(\hat{\beta})$ across the water column, for mud depths $\hat{d}=8,12$ and 16 , when the system is unbounded $(Y=0)$. To facilitate comparison, the profiles for a rigid bed or $\hat{d}=0$ are shown by dashes in these and the following figures. In an open system, the mass transport velocity increases monotonically with height above the water bed, and therefore the profile is essentially controlled by its near-bottom value. Here, we see again the depth-dependent resonance effects discussed above. At mud depth $\hat{d}=12$ (figure $4 b$ ), it is the one with elasticity $\hat{\lambda}_{e}=0.15$ that is subject to a strong amplification of the interfacial displacement, while at mud depth $\hat{d}=16$ (figure $4 c$ ), it is the one with elasticity $\hat{\lambda}_{e}=0.1$ whose interfacial 

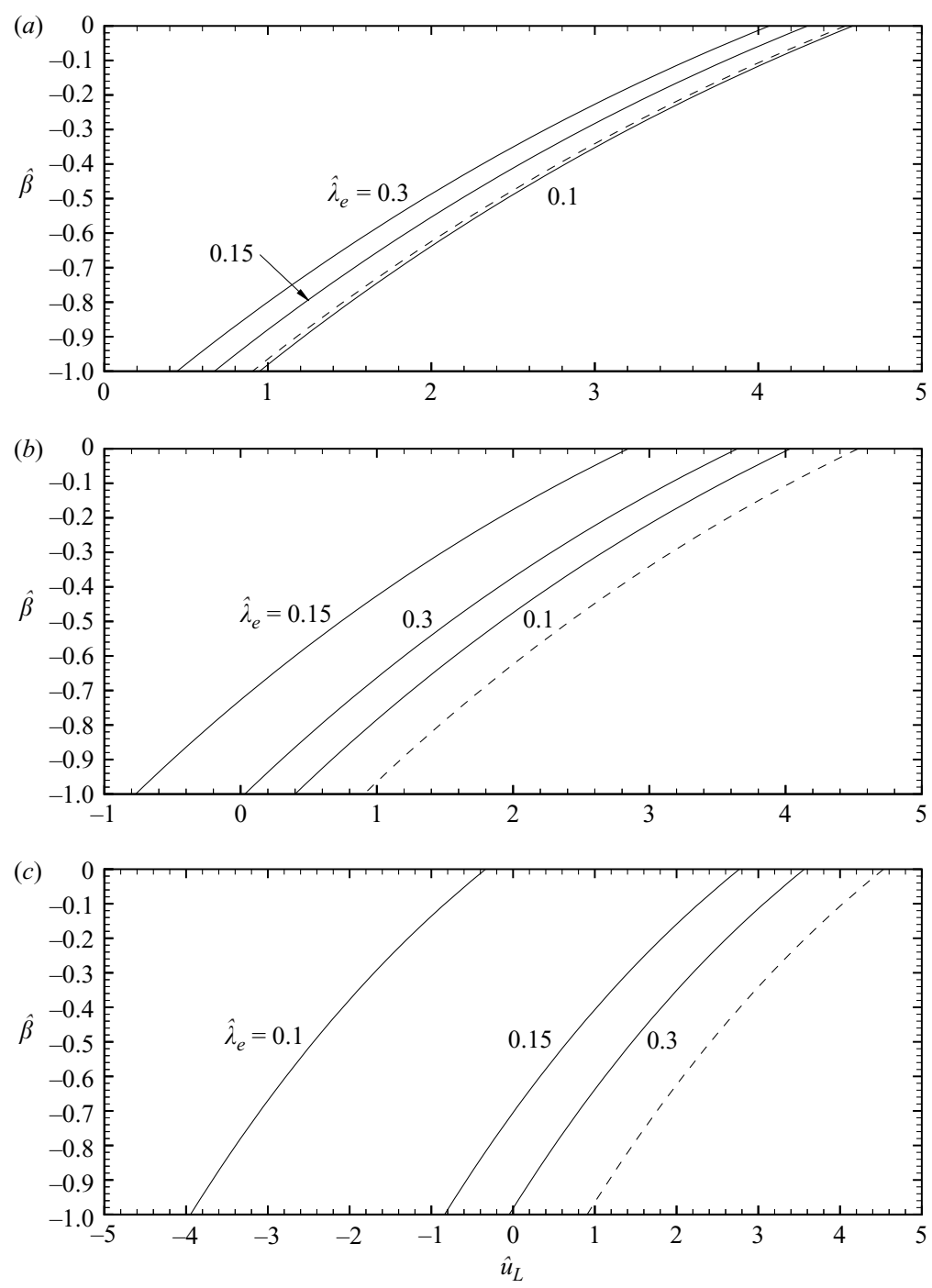

FiguRE 4. Mass transport velocity profiles $\hat{u}_{L}(\hat{\beta})$ in an open system $(Y=0)$ as a function of $\hat{\lambda}_{e}$, where $(a) \hat{d}=8,(b) \hat{d}=12,(c) \hat{d}=16$. The profiles for the limiting case of a rigid bed $(\hat{d}=0)$ are shown by dashes for comparison.

displacement is the most amplified among the cases shown. When the mud motion is sufficiently amplified, the mass transport velocity is negative immediately above the water-mud interface. As it increases with height, the mass transport velocity may turn from negative in the lower part of the water column to positive in the upper part of the water column. However, when the bottom drift velocity is strongly negative, the mass transport will remain negative across the entire water column. The overall mass transport velocity profile turns out to be very dependent on the extent of resonance amplification of the mud motion, and therefore it varies in a non-monotonic manner with the mud depth $\hat{d}$ and elasticity $\hat{\lambda}_{e}$.

The mass transport velocity profiles for the counterpart of a closed system $(Y=1)$ are shown in figure 5. Let us compare again our results with those of Wen \& Liu 

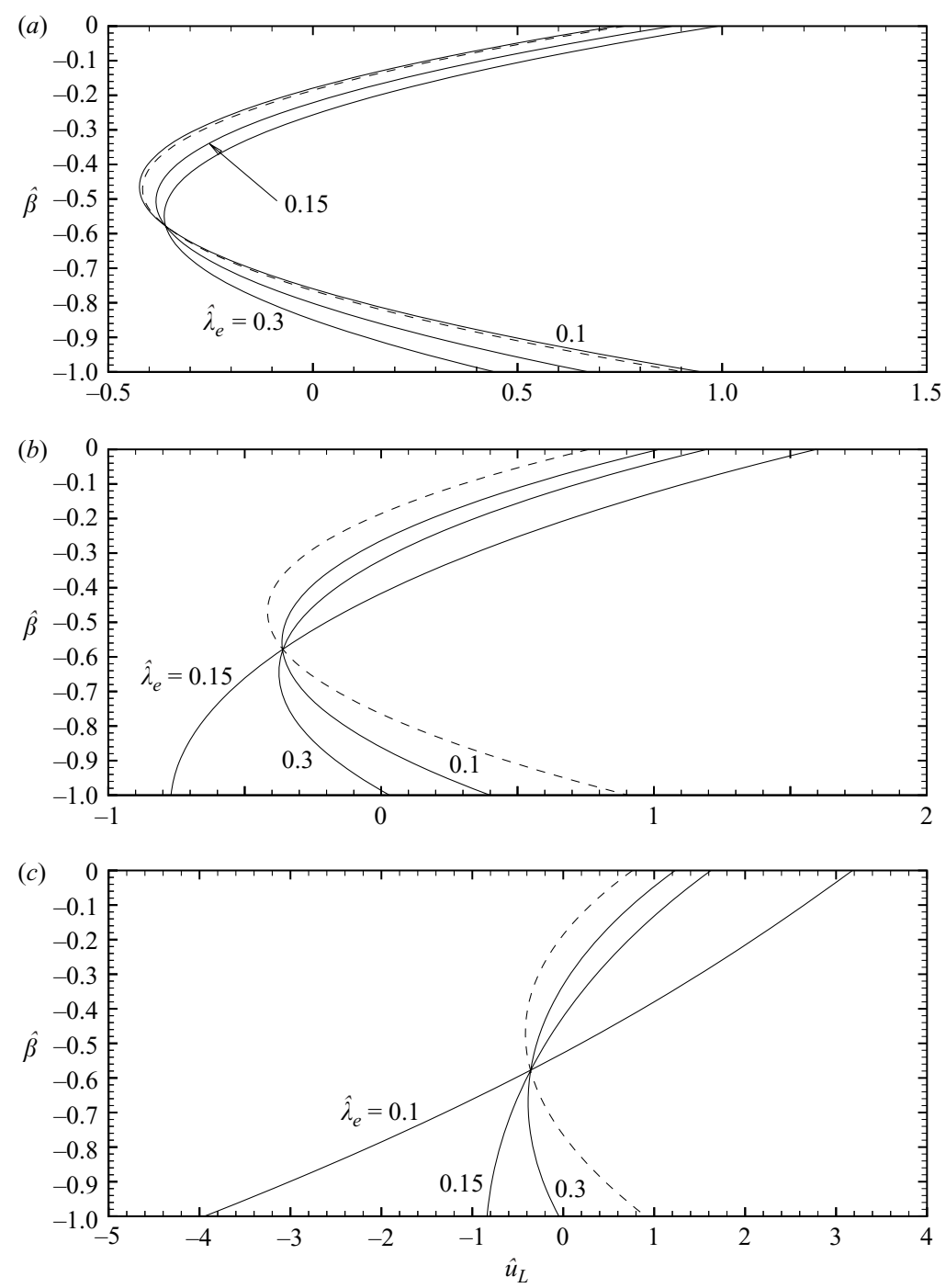

FIGURE 5. Mass transport velocity profiles $\hat{u}_{L}(\hat{\beta})$ in a closed system $(Y=1)$ as a function of $\hat{\lambda}_{e}$, where $(a) \hat{d}=8,(b) \hat{d}=12,(c) \hat{d}=16$. The profiles for the limiting case of a rigid bed $(\hat{d}=0)$ are shown by dashes for comparison.

(1995a). On considering an infinitely deep bed of strong elasticity, $G=O\left(10^{3}-10^{8}\right) \mathrm{Pa}$, Wen \& Liu found that the elastic deformation of the bed is to enhance both the forward drift in the lower part of the water column and the backward drift in the upper part of the water column. Here, we consider a thin layer of soft viscoelastic mud, and find that the opposite effect is true: the near-bed drift is decreased to the extent that it becomes reversed in direction (as has been discussed above), while the near-surface forward drift is appreciably enhanced. While Wen \& Liu (1995a) found a factor of as large as 2 in the effect of elasticity on the mass transport velocity, we here find that the mass transport velocity can be affected by a much larger factor, primarily owing to the resonance amplification of the interfacial displacement. The comparison 

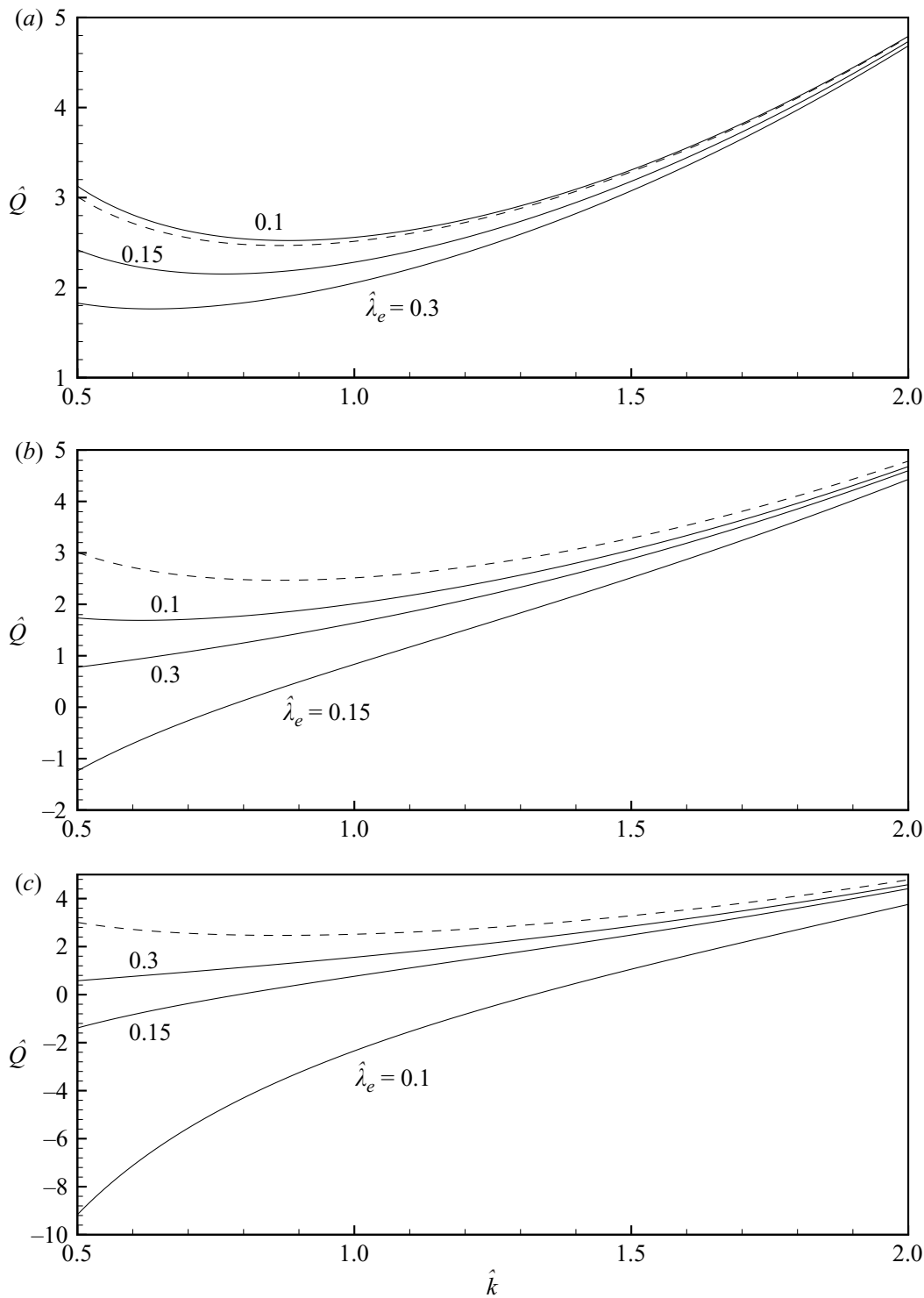

Figure 6. Net mass transport discharge $\hat{Q}$ in an open system $(Y=0)$ as a function of $\hat{k}$ and $\hat{\lambda}_{e}$, where $(a) \hat{d}=8,(b) \hat{d}=12,(c) \hat{d}=16$. The discharge for the limiting case of a rigid bed $(\hat{d}=0)$ is shown by dashes for comparison.

suggests that a thin layer of very soft bottom mud can have a greater impact than a thick layer of stiff soil deposits on the mass transport in the water column.

Finally, we show in figure 6 the net mass transport discharge $\hat{Q}$ as a function of $\hat{k}$, corresponding to the cases shown in figure 4, when the system is open. As is expected, a viscoelastic bed is to lead to a smaller forward mass flux, or even a backward flux, when compared with the flux over a rigid bed. Clearly, the effect of the bed is larger for smaller $\hat{k}$ or a shallower water layer. It is remarkable that when $\hat{k}=0.5$, the negative mass flux over an viscoelastic bed can be several times larger in magnitude 
than the forward flux over a rigid bed. The longer the wave, the greater the effect of a viscoelastic bed on the mass transport in the water layer.

\section{Concluding remarks}

We have developed analytical expressions for the mass transport in a progressive water wave over a thin layer of viscoelastic material, and shown that the results are qualitatively different from the cases when the material is purely viscous or purely elastic with infinite depth. A pure viscous muddy bottom only mildly decreases the mass transport, which remains in the same direction as the wave propagation. In sharp contrast, a viscoelastic material can lead to a significant change in the magnitude of the mass transport in the water column, and even a change in the drift direction. We further remark that mass transport over a finite viscoelastic layer can be dramatically different from that over an infinitely deep elastic bottom, since resonance can occur in the former, but not in the latter. An elastic bed of infinite depth always increases the mass transport in the water layer, while a viscoelastic bed of finite depth may increase or decrease the mass transport, depending on the depth and elasticity of the bed layer. We have explained that the particle drift in the interfacial boundary layer can be zero or negative, as a result of amplification of the horizontal oscillatory displacement of the interface due to resonance. We have also demonstrated that resonance, when occurring in the bottom layer, can play a pivotal role in determining the magnitude as well as the direction of the mass transport in the water layer, whether the system is open or closed.

The present work is limited to progressive waves over a very thin viscoelastic layer. It is possible to extend the scope of the work to include (i) partially standing waves, (ii) a viscoelastic layer comparable in thickness with the water layer, (iii) a stratified bed layer with viscoelastic properties varying as a function of depth, (iv) time-varying eddy viscosity of water in the interfacial boundary layer, (v) a sloping bottom, and so on. Most of these tasks would, however, involve considerable numerical effort.

It is also of interest to investigate the additional effects on the mass transport due to a contaminated water surface. Weber \& Christensen (2003) and Christensen (2005) have studied the mean drift induced by waves on a surface covered by a monomolecular layer of surfactant modelled as an elastic film. They found that when the film is stationary, the mean drift velocity can be in the opposite direction to the wave propagation for certain values of the film elasticity. It is worth finding out how the elasticity of a surface film interacts with the elasticity of a bottom material in determining the mean drifts in the interior of a water layer.

Comments by the three referees are gratefully acknowledged. This study constitutes part of the work performed by X.Z. for his MPhil thesis under the supervision of C.-O.N. at the University of Hong Kong. The work was supported by the Research Grants Council of the Hong Kong Special Administrative Region, China, through Project nos. HKU 7081/02E, and HKU 7199/03E.

\section{REFERENCES}

Chou, H.-T., Foda, M. A. \& Hunt, J. R. 1993 Rheological response of cohesive sediments to oscillatory forcing. In Nearshore and Estuarine Cohesive Sediment Transport, vol. 42 (ed. A. J. Mehta), pp. 126-148. AGU.

Christensen, K. H. 2005 Transient and steady drift currents in waves damped by surfactants. Phys. Fluids 17 (4), paper 042102. 
Dalrymple, R. A. \& LiU, P. L.-F. 1978 Waves over soft muds: a two-layer fluid model. J. Phys. Oceanogr. 8, 1121-1131.

Dore, B. D. 1970 Mass transport in layered fluid systems. J. Fluid Mech. 40, 113-126.

FodA, M. A., Hunt, J. R. \& Chou, H.-T. 1993 A nonlinear model for the fluidization of marine mud by waves. J. Geophys. Res. 98 (C4), 7039-7047.

HiLl, D. F. \& FoDA, M. A. 1999 Effects of viscosity and elasticity on the nonlinear resonance of internal waves. J. Geophy. Res. 104 (C4), 10951-10957.

Hsiao, S. V. \& Shemdin, O. H. 1980 Interaction of ocean waves with a soft bottom. J. Phys. Oceanogr. 10, 605-610.

Hutter, K. \& JöHnK, K. 2004 Continuum Methods of Physical Modeling. Springer.

Isobe, M., Huynh, T. N. \& Watanabe, A. 1992 A study on mud mass transport under waves based on an empirical rheology model. In Proc. 23rd Intl Conf. Coastal Engng, pp. 3093-3106.

Kirby, R. R., Hobbs, C. H. \& Menta, A. J. 1989 Fine sediment regime of Lake Okeechobee, Florida. Rep. UFL/COEL-89/009, Coastal and Oceanographic Engineering Department, University of Florida.

LaI, W. M., Rubin, D. \& Krempl, E. 1993 Introduction to Continuum Mechanics, 3rd edn. Pergamon.

LI, Y. \& Menta, A. J. 2001 Fluid mud in the wave-dominated environment revisited. In Coastal and Estuarine Fine Sediment Processes (ed. W. H. McAnally \& A. J. Mehta), pp. 79-93. Elsevier.

LiAn, J., ZhaO, Z. \& ZhANG, Q. 1999 A nonlinear viscoelastic model for bed mud transport due to waves and currents. Chinese Sci. Bull. 44, 1597-1600.

LiU, P. L.-F. 1977 Mass transport in water waves propagated over a permeable bed. Coastal Engng 1, 79-96.

Longuet-Higgins, M. S. 1953 Mass transport in water waves. Phil. Trans. R. Soc. Lond. A 245, 535-581.

MaA, J. P.-Y. \& Menta, A. J. 1988 Soft mud properties: Voigt model. J. Waterway Port Coastal Ocean Engng 114, 765-770.

MaA, J. P.-Y. \& Menta, A. J. 1990 Soft mud response to water waves. J. Waterway Port Coastal Ocean Engng 116, 634-650.

MacPherson, H. 1980 The attenuation of water waves over a non-rigid bed. J. Fluid Mech. 97, 721-742.

Mallard, W. W. \& Dalrymple, R. A. 1977 Water waves propagating over a deformable bottom. Proc. Offshore Tech. Conf. OTC 2895, pp. 141-146.

Meнта, A. J. 1996 Interaction between fluid mud and water waves. In Environmental Hydraulics (eds. V. P. Singh \& W. H. Hager), pp. 153-187. Kluwer.

MeI, C. C. 1989 The Applied Dynamics of Ocean Surface Waves. World Scientific.

MeI, C. C. \& LiU, K.-F. 1987 A Bingham-plastic model for a muddy seabed under long waves. J. Geophys. Res. 92, $14581-14594$.

Monin, A. S. \& Yaglom, A. M. 1971 Statistical Fluid Mechanics. MIT Press.

NG, C.-O. 2000 Water waves over a muddy bed: a two-layer Stokes' boundary layer model. Coastal Engng 40, 221-242.

NG, C.-O. 2004a Mass transport in gravity waves revisited. J. Geophys. Res. 109 (C4), art. no. C04012, doi:10.1029/2003JC002121.

NG, C.-O. $2004 b$ Mass transport and set-ups due to partial standing surface waves in a two-layer viscous system. J. Fluid Mech. 520, 297-325.

NG, C.-O., Fu, S. C. \& BAI, Y. C. 2002 Mass transport in a thin layer of bi-viscous mud under surface waves. China Ocean Engng 16, 423-436.

Piedra-Cueva, I. 1993 On the response of a muddy bottom to surface water waves. J. Hydraul. Res. 31, 681-696.

Piedra-Cueva, I. 1995 Drift velocity of spatially decaying waves in a two-layer viscous system. J. Fluid Mech. 299, 217-239.

Pierson, W. J. 1962 Perturbation analysis of the Navier-Stokes equations in Lagrangian form with selected linear solutions. J. Geophys. Res. 67, 3151-3160.

Rodriguez, H. N. \& MehtA, A. J. 1998 Considerations on wave-induced fluid mud streaming at open coasts. In Sedimentary Processes in the Intertidal Zone (ed. K. S. Black, D. M. Paterson \& A. Cramp), pp. 177-186, Geological Society, London, Special Publications, vol. 139.

SAKakiyama, T. \& BiJker, E. W. 1989 Mass transport velocity in mud layer due to progressive waves. J. Waterway Port Coastal Ocean Engng 115, 614-633. 
Shibayama, T., OKuno, M. \& Sato, S. 1990 Mud transport rate in mud layer due to wave action. In Proc. 22nd Coastal Engng Conf. pp. 3037-3049.

Soltanpour, M., Shibayama, T. \& Noma, T. 2003 Cross-shore mud transport and beach deformation model. Coastal Engng J. 45, 363-386.

TChen, C.-M. 1956 Interfacial waves in viscoelastic media. J. Appl. Phys. 27, 431-434.

Trowbridge, J. \& MAdSEN, O. S. 1984 Turbulent wave boundary layers 2. Second-order theory and mass transport. J. Geophys. Res. 89, 7999-8007.

ÜnlüAtA, Ü. \& MeI, C. C. 1970 Mass transport in water waves. J. Geophys. Res. 75, 7611-7618.

Verreet, G. \& Berlamont, J. 1988 Rheology and non-Newtonian behavior of sea and estuarine mud. In Encyclopedia of Fluid Mechanics, vol. 7 (ed. N. P. Cheremisinoff), pp. 135-149. Gulf.

Weber, J. E. \& Christensen, K. H. 2003 Mean drift induced by free and forced dilational waves. Phys. Fluids 15 (12), 3703-3709.

Wen, J. \& LiU, P. L.-F. 1995 a Mass transport in water waves over an elastic bed. Proc. R. Soc. Lond. A 450, 371-390.

Wen, J. \& LiU, P. L.-F. $1995 b$ Mass transport of interfacial waves in a two-layer fluid system. J. Fluid Mech. 297, 231-254.

Whitehouse, R., Soulsby, R., Roberts, W. \& Mitchener, H. 2000 Dynamics of Estuarine Muds. Thomas Telford.

WinterwerP, J. C. 1999 On the dynamics of high-concentrated mud suspensions. PhD thesis, Faculty of Civil Engineering and Geosciences, Delft University of Technology.

ZhANG, X. \& NG, C.-O. $2006 a$ Mud-wave interaction: a viscoelastic model. China Ocean Engng 20, $15-26$.

ZhANG, X. \& NG, C.-O. $2006 b$ On the oscillatory and mean motions due to waves in a thin viscoelastic layer. Wave Motion 43, 387-405. 